NASA Technical Memorandum 4655

\title{
Development of Finite Elements for Two- Dimensional Structural Analysis Using the Integrated Force Method
}

Igor Kaljević, Surya N. Patnaik, and Dale A. Hopkins

MARCH 1996

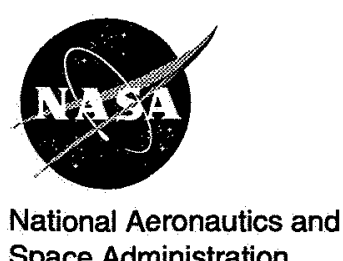

Space Administration 
NASA Technical Memorandum 4655

\section{Development of Finite Elements for Two- Dimensional Structural Analysis Using the Integrated Force Method}

Igor Kaljević and Surya N. Patnaik

Ohio Aerospace Institute

Cleveland, Ohio

Dale A. Hopkins

Lewis Research Center

Cleveland, Ohio

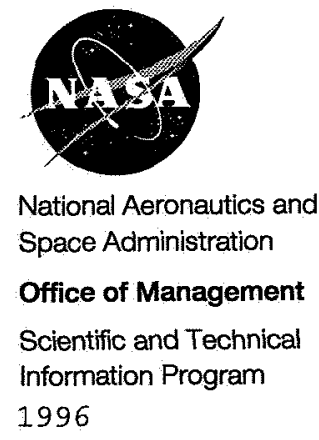




\title{
DEVELOPMENT OF FINITE ELEMENTS FOR TWO-DIMENSIONAL STRUCTURAL ANALYSIS USING THE INTEGRATED FORCE METHOD
}

\author{
Igor Kaljević and Surya N. Patnaik \\ Ohio Aerospace Institute \\ Cleveland, $\mathrm{OH} 44142$ \\ and \\ Dale A. Hopkins \\ National Aeronautics and Space Administration \\ Lewis Research Center \\ Cleveland, OH 44135
}

\section{Summary}

The Integrated Force Method has been developed in recent years for the analysis of structural mechanics problems. This method treats all independent internal forces as unknown variables that can be calculated by simultaneously imposing equations of equilibrium and compatibility conditions. In this paper a finite element library for analyzing two-dimensional problems by the Integrated Force Method is presented. Triangular- and quadrilateral-shaped elements capable of modeling arbitrary domain configurations are presented. The element equilibrium and flexibility matrices are derived by discretizing the expressions for potential and complementary energies, respectively. The displacement and stress fields within the finite elements are independently approximated. The displacement field is interpolated as it is in the standard displacement method, and the stress field is approximated by using complete polynomials of the correct order. A procedure that uses the definitions of stress components in terms of an Airy stress function is developed to derive the stress interpolation polynomials. Such derived stress fields identically satisfy the equations of equilibrium. Moreover, the resulting element matrices are insensitive to the orientation of local coordinate systems. A method is devised to calculate the number of rigid body modes, and the present elements are shown to be free of spurious zero-energy modes. A number of example problems are solved by using the present library, and the results are compared with corresponding analytical solutions and with results from the standard displacement finite element method. The Integrated Force Method not only gives results that agree well with analytical and displacement method results but also outperforms the displacement method in stress calculations.

\section{Introduction}

The finite element stiffness method, which is based on an assumed displacement field, has become the method of choice for solving a wide variety of problems in structural mechanics. The advantages of the stiffness method include (1) the capability to efficiently and accurately model domains with complex geometric configurations and varying material properties and (2) the capability to accurately analyze problems with geometrical and material nonlinearities. The development of finite stiffness elements and their corresponding formulations has been a subject of extensive research, much of which has been summarized in textbooks such as references 1 to 3 .

Shortcomings of the assumed displacement method have been observed in the analyses of certain classes of problems, such as modeling nearly incompressible materials, bending of thin plates, and optimizing structures (refs. 4 and 5). Moreover, since stresses are calculated indirectly by using displacement derivatives, the accuracy of stress predictions may be reduced. Two alternative finite element formulations may be utilized to analyze the aforementioned problems and to calculate stress more accurately: (1) the hybrid stress method (refs. 6 to 8), and (2) the force method (refs. 9 to 11). Because both of these formulations have certain disadvantages compared to the assumed displacement method, their use and availability in general purpose programs has been limited. In the hybrid method, the flexibility matrix must be inverted in order to generate the element stiffness matrix; this can become a computational burden, especially if high order approximations of stress fields are required. In the standard force method, on the other hand, an auxiliary statically determinate structure and a corresponding set of redundant forces must be selected. This 
process is not easily adapted to computer automation. Several attempts have been made to improve the process by which redundancies are selected. The pertinent formulations were summarized by Kaneko et al. (ref. 5). All of these procedures, however, either resulted in matrices with certain undesired properties or lacked a physical interpretation, which made them unattractive to the engineering community and led to the demise of the standard force method.

An alternateformulation, termed the Integrated Force Method, has been developed in recent years to analyze problems in structural mechanics (refs. 12 to 15). In the Integrated Force Method all independent forces, not just the redundants, are treated as unknown quantities that can be calculated by simultaneously imposing both equilibrium and compatibility conditions. Procedures have been developed (refs. 16 to 19) for generating compatibility conditions that yield sparse and banded matrices and can be easily adapted to computer automation. The initial applications of the Integrated Force Method to static analysis (ref. 20), vibration analysis (ref. 21), and optimization of trusses (ref. 22) have shown that the Integrated Force Method has certain advantages over the displacement method, both in accuracy and computer efficiency.

This study presents formulations to develop finite elements for two-dimensional structural analysis and a comprehensive finite element library of two-dimensional elements. Both triangular- and quadrilateral-shaped elements capable of modeling arbitrary configurations of the domains being analyzed are considered. The displacement and stress fields within an element are independently approximated. The displacement field is interpolated by using the functions employed in the standard displacement method. Stress fields are approximated by using complete polynomials of the appropriate order, whose coefficients are unknown independent forces. The equations describing the components of the stress tensor can be derived from the Airy stress function for an element, which is written in terms of a complete polynomial of a certain order. The resulting stress fields identically satisfy the equations of equilibrium. The element matrices generated with these stress fields are not sensitive to the orientation of the element's local coordinate system. A method to calculate the number of zeroenergy modes is also developed, and the present elements are shown to be free of spurious zero-energy modes. The effect of reducing the number of the element's independent forces is also investigated.

To establish the validity of the elements and to assess their relative performances and compare the Integrated Force Method with the well established displacement method, the present library is used to solve a variety of problems in two-dimensional elasticity. The results obtained with these elements are also compared with the corresponding analytical solutions, and there is good agreement. For stress calculations, the Integrated Force Method performs better than the standard displacement method.

\section{Development of the Finite Elements}

\section{Governing Equations of the Integrated Force Method}

The governing equations of the Integrated Force Method are briefly presented here in order to introduce the notation. Symbols used are defined in appendix A. (A detailed description of the formulation can be found in refs. 12 to 14.) Finite elements are used to discretize a continuous object, which then has $N_{t}$ displacement degrees of freedom and $m$ independent forces. In the Integrated Force Method all independent forces represent unknown quantities, not just the redundants, as is the case in the standard force method (refs. 9 to 11). The unknown forces are obtained from the following sets of equations:

$$
\left[\mathbf{B}_{u}\right]\{\mathbf{F}\}=\{\mathbf{P}\}
$$

which represents $n$ equations of equilibrium, written for nodes where displacements are unknown, and

$$
[\mathbf{C}][\mathbf{G}][\mathbf{F}\}=\left\{\delta_{o}\right\}
$$

which represents $r=m-n$ compatibility conditions.

Here $n=N_{t}-N_{s} ; N_{s}$ is the number of prescribed displacement degrees of freedom; $\{\mathbf{F}\}$ is the $m$-component vector of unknown independent forces; $\left[\mathbf{B}_{u}\right]$ is the $n \times m$ part of the system equilibrium matrix corresponding to the nodes where external loads are prescribed; [G] is the $m \times m$ system flexibility matrix; $[\mathbf{C}]$ is the $r \times m$ compatibility matrix; $\{\mathbf{P}\}$ is the $n$-component vector of equivalent nodal loads; and $\left\{\delta_{o}\right\}$ is the $r$-component effective deformation vector, which is calculated as

$$
\left\{\delta_{o}\right\}=-[\mathbf{C}]\left\{\beta_{o}\right\}
$$

where $\left\{\beta_{o}\right\}$ is the vector of initial deformations. The generation of compatibility conditions is described in detail in references 16 to 19 . Sets of equations (1) and (2) can be combined to obtain the system of equations for unknown forces as

$$
[\mathbf{S}]\{\mathbf{F}\}=\left\{\mathbf{P}^{*}\right\}
$$

where

$$
[\mathbf{S}]=\left[\begin{array}{c}
{\left[\mathbf{B}_{u}\right]} \\
{[\mathbf{C}][\mathbf{G}]}
\end{array}\right] \text { and }\{\mathbf{P} *\}=\left\{\begin{array}{c}
\{\mathbf{P}\} \\
\left\{\delta_{o}\right\}
\end{array}\right\}
$$

After the force vector is calculated from equation (4), the vector of unknown nodal displacements $\{\mathbf{U}\}$ can be obtained as

$$
\{\mathbf{U}\}=[\mathbf{J}][\mathbf{G}]\{\mathbf{F}\}
$$


where $[\mathbf{J}]$ is the $n \times m$ deformation matrix that represents the top $n$ rows of the transpose of the matrix $[\mathbf{S}]^{-1}$. The vector of unknown support reactions $\{\mathbf{R}\}$ can be calculated from

$$
\{\mathbf{R}\}=\left[\mathbf{B}_{s}\right]=\{\mathbf{F}\}
$$

where $\left[\mathbf{B}_{s}\right]$ is the portion of the system equilibrium matrix that corresponds to nodes with prescribed displacement boundary conditions.

\section{Element Matrices}

From equations (1) and (2) we see that the system of equations for the unknown forces consists of two sets of relations: (1) equations of equilibrium and (2) compatibility conditions, which can be expressed in terms of forces by using the strain-stress law. These sets of relations are first established on the element level, and then the assembly procedure (refs. 13 and 14) is used to derive the system given in equation (4).

Equilibrium equations and deformation-force relations for a finite element may be written as

$$
\begin{array}{r}
\left\{\mathbf{P}_{e}\right\}=\left[\mathbf{B}_{e}\right]\left\{\mathbf{F}_{e}\right\} \\
\left\{\boldsymbol{\beta}_{e}\right\}=\left[\mathbf{G}_{e}\right]\left\{\mathbf{F}_{e}\right\}
\end{array}
$$

where $\left\{\mathbf{P}_{e}\right\}$ is the vector of equivalent nodal forces for the element $e ;\left\{\mathbf{F}_{e}\right\}$ is the element vector of independent forces; $\left[\mathbf{B}_{e}\right]$ and $\left[\mathbf{G}_{e}\right]$ are element equilibrium and flexibility matrices, respectively; and $\left\{\beta_{e}\right\}$ is the vector of element deformations. Note that equation $(8 \mathrm{~b})$ represents the discretized constitutive relations for the element. The components of the vector $\left\{\beta_{e}\right\}$ are the generalized deformations that correspond to internal forces $\left\{\mathbf{F}_{e}\right\}$.

The expressions for the two element matrices can be derived by using the expressions for potential and complementary energy, respectively. In the Integrated Force Method, independent displacement and stress interpolations are employed to give

$$
\begin{aligned}
& \{\mathbf{u}\}=[\mathbf{N}]\left\{\mathbf{U}_{e}\right\} \\
& \{\sigma\}=[\mathbf{Y}]\left\{\mathbf{F}_{e}\right\}
\end{aligned}
$$

Here $\{\mathbf{u}\}^{\mathrm{T}}=\{u \nu\}$ and $\{\sigma\}^{\mathrm{T}}=\left\{\sigma_{x} \sigma_{y} \tau_{x y}\right\}$ are the displacement and stress vectors at a location within the element; $\left\{\mathbf{U}_{e}\right\}$ is the vector of element nodal displacements; $[\mathbf{N}]$ is the matrix of displacement interpolation functions; and $[\mathbf{Y}]$ is the stress interpolation matrix. The strain vector, $\{\varepsilon\}^{\top}=\left\{\varepsilon_{x} \varepsilon_{y} \gamma_{x y}\right\}$, is obtained by differentiation of the displacement field and is given as

$$
\{\varepsilon\}=[\mathbf{Z}]\left\{\mathbf{U}_{e}\right\}
$$

where $[\mathbf{Z}]=[\mathbf{L}][\mathbf{N}]$, and $[\mathbf{L}]$ is the matrix of differential operators.

Equilibrium matrix. - The expression for the element equilibrium matrix can be obtained from the strain energy $A_{p}$ of the element:

$$
A_{p}=\frac{1}{2} \int_{V}\{\varepsilon\}^{\top}\{\sigma\} d V
$$

where $V$ denotes the domain of the element in the discrete form. Substituting equations (10) and (11) into equation (12) yields the strain energy $A_{p}$ expressed as

$$
A_{p}=\frac{1}{2}\left\{\mathbf{U}_{e}\right\}^{\top}\left[\mathbf{B}_{e}\right]\left\{\mathbf{F}_{e}\right\}
$$

where the equilibrium matrix $\left[\mathbf{B}_{e}\right]$ is

$$
\left[\mathbf{B}_{e}\right]=\int_{V}[\mathbf{Z}]^{\top}[\mathbf{Y}] d V
$$

Flexibility matrix.-The expression for the element flexibility matrix can be obtained from the complementary energy $A_{c}$ of the element:

$$
A_{c}=\frac{1}{2} \int_{V}\{\sigma\}^{\top}[\mathbf{D}]\{\sigma] d V
$$

where [D] is the compliance matrix of the material, in the discrete form. Substituting equation (10) into equation (15) gives $A_{c}$ as

$$
A_{c}=\frac{1}{2}\left\{\mathbf{F}_{e}\right\}^{\top}\left[\mathbf{G}_{e}\right]\left\{\mathbf{F}_{e}\right\}
$$

where the element flexibility matrix $\left[\mathbf{G}_{e}\right]$ is

$$
\left[\mathbf{G}_{e}\right]=\int_{V}[\mathbf{Y}]^{\mathbf{T}}[\mathbf{D}][\mathbf{Y}] d V
$$

\section{Stress Field Approximations}

The approximation of the stress components and the construction of the stress interpolation matrix $[\mathbf{Y}]$ is discussed in this section. Note that in equations (14) and (17) the matrix [Y] appears in both the equilibrium and the flexibility matrix definitions. It is, therefore, important to properly devise stress interpolation polynomials in order to obtain accurate results. In 
this study, a method was developed that uses an Airy stress function given in terms of complete polynomials.

The Airy stress function $\Phi$ for a location $(x, y)$ within an element can be written as a complete polynomial of order $p$ :

$$
\Phi(x, y)=\sum_{j=0}^{p} C_{j} x^{p-j} y^{j}
$$

where $C_{j}$, for $j=0,1,2, \ldots, p$, are constants, and $x$ and $y$ are Cartesian coordinates of the point in the local coordinate system of the element. The local coordinate systems for various element shapes are depicted in figure 1 . The components of the stress tensor can be obtained by using the definition of the stress function (ref. 23) as follows:

$$
\begin{gathered}
\sigma_{x}=\sum_{j=0}^{p-2} C_{j+2}(j+1)(j+2) x^{p-2-j} y^{j} \\
\sigma_{y}=\sum_{j=0}^{p-2} C_{j}(p-j)(p-j-1) x^{p-2-j} y^{j} \\
\tau_{x y}=-\sum_{j=0}^{p-2} C_{j+1}(j+1)(p-j-1) x^{p-2-j} y^{j}
\end{gathered}
$$

\begin{tabular}{|c|c|c|c|}
\hline Shape & Nodes & Forces & Name \\
\hline & \multirow{3}{*}{3} & 3 & TRI03_03 \\
\hline & & 5 & TRI03_05 \\
\hline & & 7 & TRI03_07 \\
\hline & \multirow{3}{*}{6} & 9 & TRI06_09 \\
\hline & & 11 & TRI06_11 \\
\hline & & 12 & TRI06_12 \\
\hline & \multirow{3}{*}{4} & 5 & QUA04_05 \\
\hline & & 7 & QUA04_07 \\
\hline & & 12 & QUA04_12 \\
\hline & \multirow{3}{*}{8} & 13 & QUA08_13 \\
\hline & & 15 & QUA08_15 \\
\hline & & 18 & QUA08_18 \\
\hline
\end{tabular}

Figure 1.-Finite element library for Integrated Force Method.
Rewriting equations (19) yields

$$
\begin{gathered}
\sigma_{x}=\sum_{j=0}^{p-2} \tilde{F}_{j+1} x^{p-j-2} y^{j} \\
\sigma_{y}=\sum_{j=0}^{p-2} \tilde{F}_{j+p-1} x^{p-j-2} y^{j} \\
\tau_{x y}=\sum_{j=0}^{p-2} \tilde{F}_{j+2 p-1} x^{p-j-2} y^{j}
\end{gathered}
$$

Now the coefficients of the polynomials in equations (20) can be considered element forces $\tilde{F}_{i}$, for $i=1,2, \ldots, 3(p-1)$, and $\tilde{F}_{i}$ can be expressed in terms of $(p+1)$ constants $C_{j}$ :

$$
F_{i}=\phi_{i}\left(C_{0}, C_{1}, \ldots, C_{p}\right) \text { for } i=1,2, \ldots, 3(p-1)
$$

where $\phi_{i}$ are linear functions of constants $C_{j}$. Thus, not all forces $\tilde{F}_{i}$ are linearly independent. Final stress field interpolation polynomials can be obtained by eliminating the dependent forces; this results in $(p+1)$ independent forces when the stress function $\Phi$ is written as a complete polynomial of order $p$. Such stress fields are complete polynomials of order $(p-2)$. Expressions for the stress fields used in this study are provided in appendix B.

The preceding procedure can be demonstrated by deriving the linear terms. For this case, the stress function is represented as a complete cubic polynomial:

$$
\Phi(x, y)=C_{0} x^{3}+C_{1} x^{2} y+C_{2} x y^{2}+C_{3} y^{3}
$$

Substituting $p=3$ into equations (19) yields the following expressions for the stress components:

$$
\begin{aligned}
& \sigma_{x}^{(1)}=2 C_{2} y+6 C_{3} y=\tilde{F}_{1} x+\tilde{F}_{2} y \\
& \sigma_{y}^{(1)}=6 C_{0} x+2 C_{1} y=\tilde{F}_{3} x+\tilde{F}_{4} y \\
& \tau_{x y}^{(1)}=-2 C_{1} x-2 C_{2} y=\tilde{F}_{5} x+\tilde{F}_{6} y
\end{aligned}
$$

where the superscript 1 denotes the linear terms in the stress polynomials. From equations (23) we see that six coefficients $\tilde{F}_{i}$ are expressed in terms of four independent constants 
$C_{j}$; thus, only four $\tilde{F}_{i}$ forces are linearly independent. By eliminating two forces from equations (23), we have obtained the linear stress terms given in equations (B2). The constant, quadratic, and cubic terms can be derived similarly, and the corresponding stress terms are given in equations (B1), (B3), and (B4), respectively. Stress field representations in terms of a complete polynomial of order $p$ can be obtained by combining the expressions for orders $0,1,2, \ldots, p$. The resulting cubic stress field interpolation, which is given in equations (B5), contains 18 independent forces. The constant stress field can be obtained by retaining the first 3 forces in equations (B5); for the linear interpolation, the first 7 forces are retained, and for the quadratic interpolation, the first 12 forces are retained. The stress fields given in equations (B1) to (B4) identically satisfy the equations of equilibrium at any point inside the domain of the element. The resulting element matrices have the correct rank for arbitrary orientation of the element's local coordinate axes. They are also invariant with respect to coordinate transformation (ref. 4).

Approximations with complete polynomials may yield individual elements with a large number of independent forces. Moreover, as Spilker and Singh (ref. 24) observed, in hybrid method applications, high order stress field approximations may lead to overly rigid models. Thus, it may be necessary to reduce the number of independent forces in stress field representations while preserving all the desired properties of the resulting element matrices. The compatibility condition

$$
\nabla^{2}\left(\sigma_{x}+\sigma_{y}\right)=0
$$

(suggested in ref. 24) can be imposed to reduce the number of independent forces. Note that equation (24) is identically satisfied for stress fields represented by zero- and first-order polynomials. By applying equation (24) to quadratic and cubic terms, we obtain reduced quadratic and cubic polynomials, as given in equations (B6) and (B7), respectively.

\section{Spurious Zero-Energy Modes}

The stress fields given in equations (B1) to (B7) were derived without any reference to the shape or the number of kinematic degrees of freedom of a considered element. The number of independent forces, however, could not be chosen arbitrarily. The number of kinematic degrees of freedom $n_{e}$ and the number of independent forces $m_{e}$ for element $e$ must satisfy the relation $m_{e} \geq n_{e}-l$ (refs. 25 and 26), where $l$ is the number of rigid body modes of the element. Pian and Chen (ref. 26) showed, however, that in the application of the hybrid method this condition is only necessary, not sufficient, for the element matrices to have the correct rank. They also devised a technique, based on energy considerations, to detect spurious zero-energy modes, and they developed a means to suppress them. Spilker et al. (ref. 4) have shown that approximating stresses with complete polynomials of the appropriate order produces the correct rank. The methodology of Pian and Chen is used herein to show that in the IntegratedForce Method the correct rank of the element equilibrium matrix ensures the absence of spurious zero-energy modes.

The expression for internal energy $A_{c}$ can be rewritten by substituting $[D]\{\sigma\}=\{\varepsilon\}$ into equation (15) to obtain

$$
A_{c}=\frac{1}{2} \int_{V}\{\sigma\}^{\top}\{\varepsilon\} d V
$$

Substituting equations (10) and (11) into equation (25) gives the internal energy written as

$$
A_{c}=\frac{1}{2}\{\mathbf{F}\}^{\mathrm{T}}[\mathbf{B}]^{\mathrm{T}}\{\mathbf{U}\}
$$

From equation (26) we can see that if the element equilibrium matrix has the correct rank, there are only $l$ zero-energy modes present that correspond to rigid body modes of the element. Thus, spurious zero-energy modes can be eliminated by constructing stress fields such that the resulting equilibrium matrix has the rank $n_{r} \geq n_{e}-1$.

\section{Element Library}

Stress fields derived in previous sections can now be used to develop a comprehensive finite element library for twodimensional stress analysis by the Integrated Force Method. Let us consider both triangular and quadrilateral elements. Isoparametric functions (ref. 27) can be employed in equation (9) for both types of elements. For stress field approximations, the element's local coordinate systems $O x y$ can be defined such that the origin coincides with the centroid of the element, and the local coordinate axes are parallel to the global axes. Such an orientation avoids rotation of the coordinate systems, saves CPU time, and does not affect the response when stress fields with complete polynomial approximation are used.

The element library is depicted in figure 1. It includes two elements developed by Nagabhushanam (J. Nagabhushanam, Indian Institute of Science, Bangalore, India, personal communication, 1992) and an element suggested by Pian (ref. 6), which has four nodes and five independent forces. These elements are also implemented for comparison purposes. The element names employed here consist of three parts: the first three characters describe the shape of the element, the next two digits denote the number of element nodes, and finally, the number following the underscore indicates the number of independent forces used in the interpolation of the stress field. Features of the present elements are enumerated in the following sections.

Three-node triangular elements: TRI03_03, TRI03_05, and TRI03_07.-Three-node triangles have six displacement degrees of freedom; thus three independent forces are necessary 
to ensure the correct rank of element matrices. Three different stress fields were implemented. The constant stress field was used for element TRI03_03. A complete linear stress field was implemented for element TRI03_07. And the stress field given in equations (B8), which is used extensively in the hybrid method (refs. 6 and 11), was used for element TRI03_05. Note that element TRI03_03 contains the minimum number of independent forces. Such elements will be referred to as statically determinate elements.

Six-node triangular elements: TRI06_09, TRI06_11, and TRI06_12.-Six-node elements have 12 displacement degrees of freedom; thus 9 independent forces are necessary in the stress field approximation. This allows quadratic polynomials to be used for stress interpolations. Complete quadratic polynomials with 12 independent forces were used for element TRI06_12, and reduced quadratic polynomials with 11 independent forces were used for element TRI06_11. For comparison the stress field suggested by Nagabhushanam (Indian Institute of Science, Bangalore, India, personal communication, 1992; see eqs. (B9)) was implemented for element TRI06_09. This stress field is represented by incomplete second-order polynomials; however, it identically satisfies the equations of equilibrium and does not possess spurious zero-energy modes.

Four-node quadrilateralelements:QUA04_05,QUA04_07, and QUA04_12.-Four-node elements have eight displacement degrees of freedom; thus five forces are necessary in the stress field approximation. The five-force field given in equations (B8) was implemented for element QUA04_05 and resulted in a statically determinate four-node element. A complete linear polynomial was implemented for element QUA04_07, and a complete quadratic polynomial was used for element QUA04_12. The equations of equilibrium were identically satisfied for all elements and no spurious zero-energy modes were detected.

Eight-node quadrilateral elements:QUA08_13,QUA08_15, and QUA08_18.-Because eight-node elements have 16 displacement degrees of freedom, they require at least 13 independent forces to approximate the stress field. Quadratic polynomials do not contain a sufficient number of terms, so cubic polynomials must be used. Complete cubic polynomials (see eqs. (B5)) were used for element QUA08_18, and reduced polynomials were used for element QUA08_15. These elements were insensitive to rotation of the coordinate axes and did not possess spurious zero-energy modes. The stress field suggested by Nagabhushanam (Indian Institute of Science, Bangalore, India, personal communication, 1992; see eqs. (B10)) was also implemented for element QUA08_13. A quadratic field that did not satisfy the equations of equilibrium resulted. It also possessed two spurious zero-energy modes.

For all elements presented here, numerical integration was used to calculate the element matrices. In the case of the triangular elements, one-point integration was used for element TRI03_03, the three-point rule was used for elements with linear interpolation of the stress field, and the seven-point rule was used for elements with quadratic interpolation of the stress field. The locations of integration points were taken from reference 28 . In the case of quadrilateral elements, standard Gauss integration was employed, with the $2 \times 2$ rule for elements with linear approximations of the stress field, the $3 \times 3$ rule for elements with quadratic approximations, and the $4 \times 4$ rule for elements with cubic approximations of the stress field.

A patch test was performed for the present elements. Stress boundary conditions were prescribed for a finite element model of the test problem taken from reference 29 , and all elements from the present library passed the patch test.

\section{Numerical Examples}

A number of example problems are presented in this section. Extensive numerical experiments were performed in order to establish the validity and accuracy of the Integrated Force Method, as well as to assess the relative performance of the present elements. The results obtained with present developments are compared herein with corresponding analytical solutions. For some problems the responses obtained from the standard displacement method are also given in order to assess the potential advantages of the Integrated Force Method. The eight-node isoparametric element (ref. 27) was used in all displacement method calculations.

\section{Example 1: Bending of a Uniform Cantilever Beam}

Consider a cantilever beam of length $L$ and uniform rectangular cross section $d$ by $H$, as șown in figure 2(a). Assume that the beam is subjected to two distinct load cases: (1) a concentrated force of intensity $P$, and (2) a uniformly distributed load of intensity $q$, and assume that the beam is made of a homogeneous and isotropic material with a modulus of elasticity $E$ and Poisson's ratio v. By using two-dimensional finite element discretizations and assuming a state of plane stress, we can analyze the response of the beam. Forthis case the entire element library was implemented in order to establish the relative performance of the present elements.

The influence of element shapes on the results was also investigated. Finite element discretizations obtained by using quadrilateral- and triangular-shaped elements are shown in figure 2, parts (b) and (c), respectively. The support conditions for modeling the beam with a clamped end assumed point $a$ to be completely fixed and the horizontal displacements at points $b$ and $c$ to be suppressed. The circles in parts (b) and (c) of figure 2 denote corner nodes, and the asterisks denote midside nodes. In discretizations using three-node triangular elements and four-node quadrilateral elements, midside nodes are not present. The dashed lines in figure 2(b) represent quadrilateral elements of distorted shapes. The concentrated force $P$ was modeled by using nodal forces of intensities $P_{1}$ and $\dot{P}_{2}$ and 
(a)

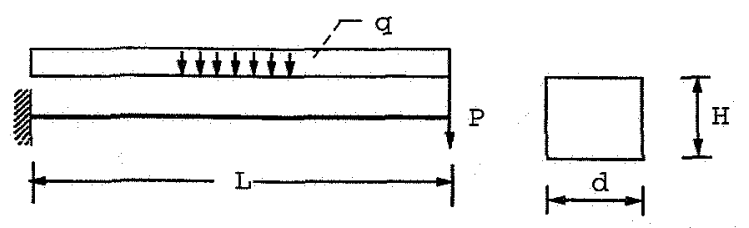

(b)
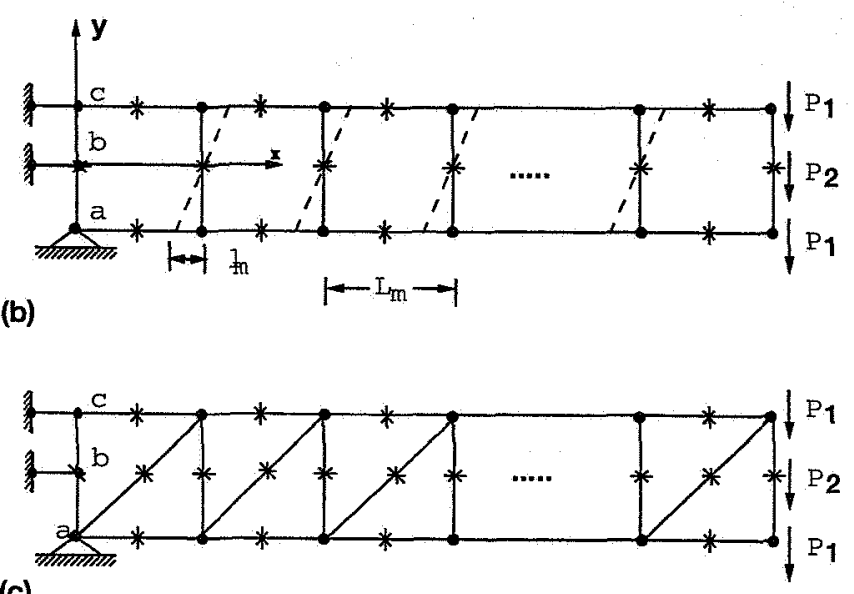

(c)

Figure 2.-A uniform cantilever beam showing geometric characteristics, loadings, and two-dimensional finite element models. (a) Subjected to concentrated force $P$ and uniformly distributed load q. (b) Discretization using quadrilateral elements. (c) Discretization using triangular elements.

assuming a parabolic distribution of the shear stress along the free end; the results were $P_{1}=0.5 P$, for elements with linear interpolation of geometry; and $P_{1}=0.1 P$ and $P_{2}=0.8 P$, for elements with quadratic interpolation of geometry.

To analyze the beam for load case (1), convergence of the tip displacement was studied for discretizations by using various numbers of elements; the results are shown in figure 3 for triangular elements and in figure 4 for quadrilateral elements. The tip displacements were normalized with respect to the exact solution from the beam theory, including the average effect of shear stresses. Figure 3 shows that three-node triangular elements lead to very slow convergence of tip displacement, whereas sixnode triangles provide accurate results with a relatively small number of elements and corresponding independent forces. The results shown in figure 3 also reveal that increasing the order of the stress approximation for three-node triangles does not improve the accuracy.

Tip displacement convergence of quadrilateral elements was first studied with discretizations using elements of rectangular shape. The results, presented in figure 4(a), show that all eightnode elements provided accurate results with a relatively small number of independent forces. Element QUA08_13, however, led to spurious zero-energy modes. Some additional degrees of freedom had to be suppressed in order to obtain a stable structure. For the present analysis, vertical displacements at points

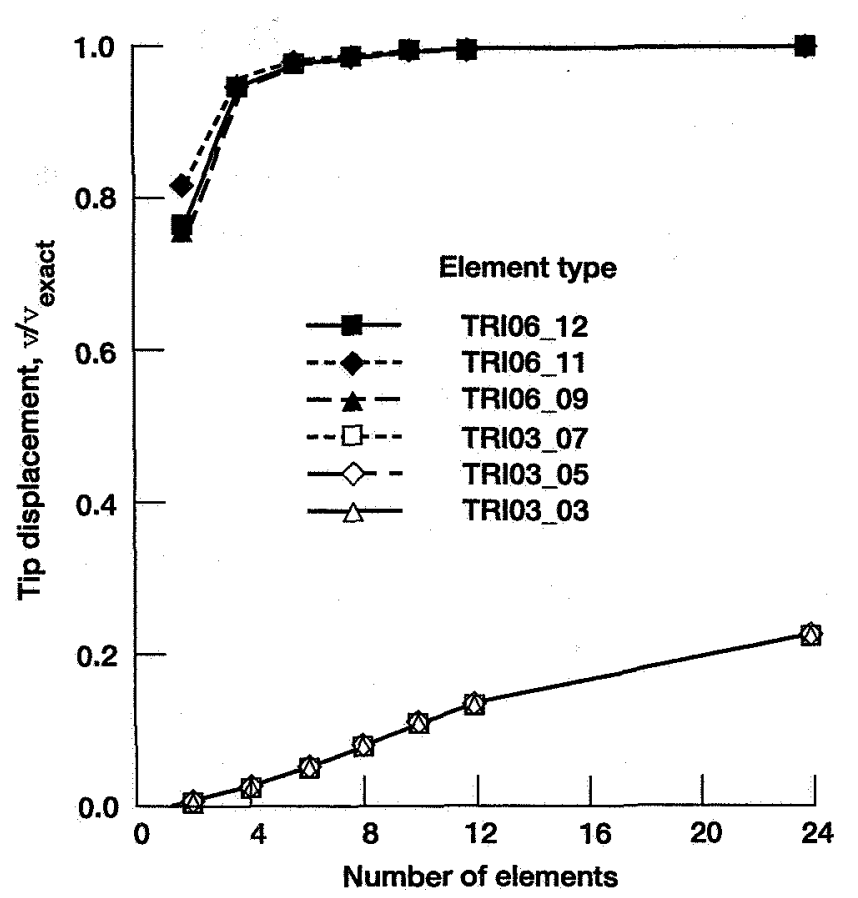

Figure 3.-Convergence study of tip displacement of cantilever beam using triangular elements.

$b$ and $c$ were set to zero. These additional restraints resulted in convergence in the opposite direction for element QUA08_13. Figure 4(a) also shows the results for four-node quadrilateral elements. Element QUA04_05 provided a fast convergence, whereas elements QUA04_07 and QUA04_12, which use higher order stress approximations, produced stiff structures. Note, however, that both of these elements provide results within 0.5 -percent error for a 24-element model.

The effect that distortion of the element shapes has on the results was also studied. The distorted meshes were obtained by moving the corner nodes a distance of $l_{m}=0.2 L_{m}$ as shown in figure 2(b), where $L_{m}$ is the length of the corresponding rectangular element. The results (see fig. 4(b)) show that fournode elements, especially element QUA04_05, are significantly less accurate. The eight-node elements, however, are almost insensitive to distortion in the model.

Now let us consider stress distributions on the beam for load case (2). The intensity of the distributed load is taken as $q=12 \mathrm{kN} / \mathrm{m}$; the length as $L=12 \mathrm{~m}$; the cross section dimensions as $d=H=1.0 \mathrm{~m}$; and Poisson's ratio as $v=0.3$. The values for normal stresses $\sigma$ and shear stresses $\tau$ along the line $y=-y_{g}=-0.2887 \mathrm{~m}$, which were obtained by using eight-node quadrilateral elements, are shown in figure 5 along with those calculated by using the beam theory. The locations shown in figure 5 coincide with the Gauss points for the $2 \times 2$ integration rule, which have been shown to be optimal sampling points for stress calculations in the displacement and hybrid methods (ref. 4). Figure 5 shows there is good agreement between the present results and the analytical solution and that results 

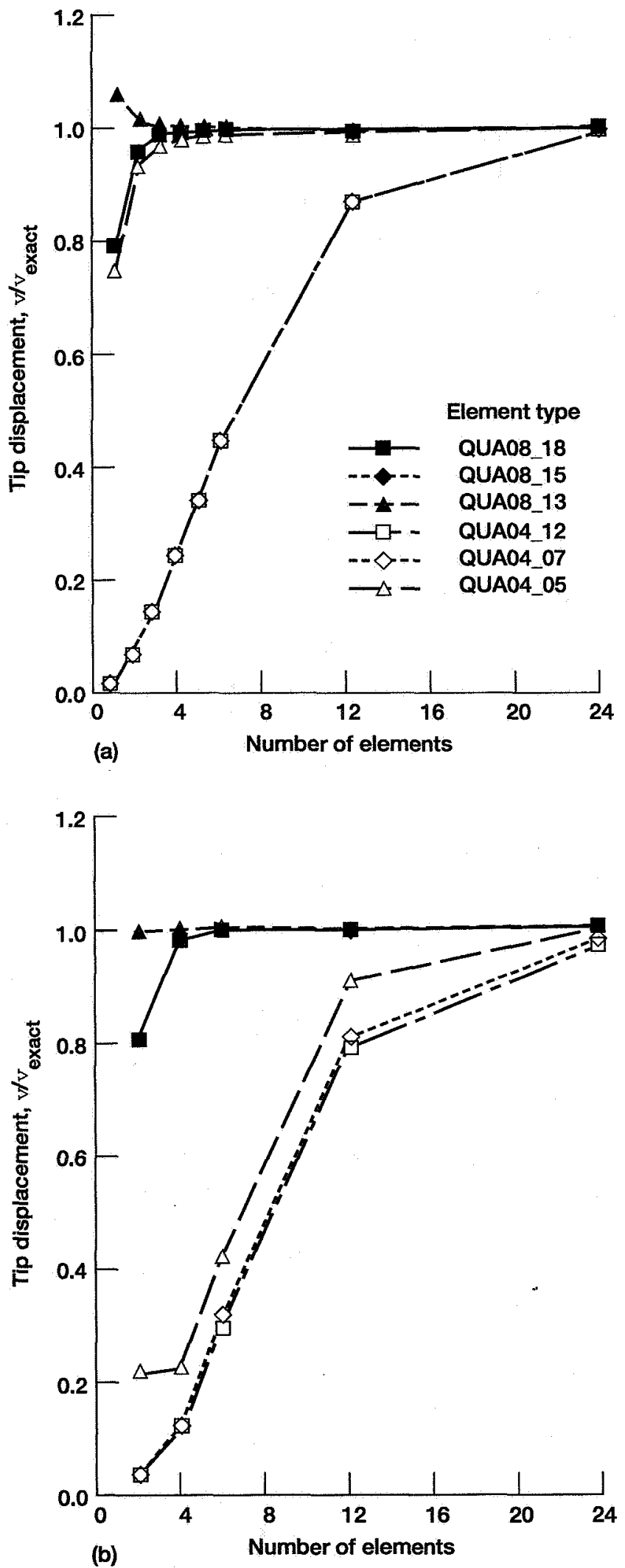

Figure 4.-Convergence study of tip displacement of cantilever beam using quadrilateral elements. (a) Regular meshes. (b) Distorted meshes.
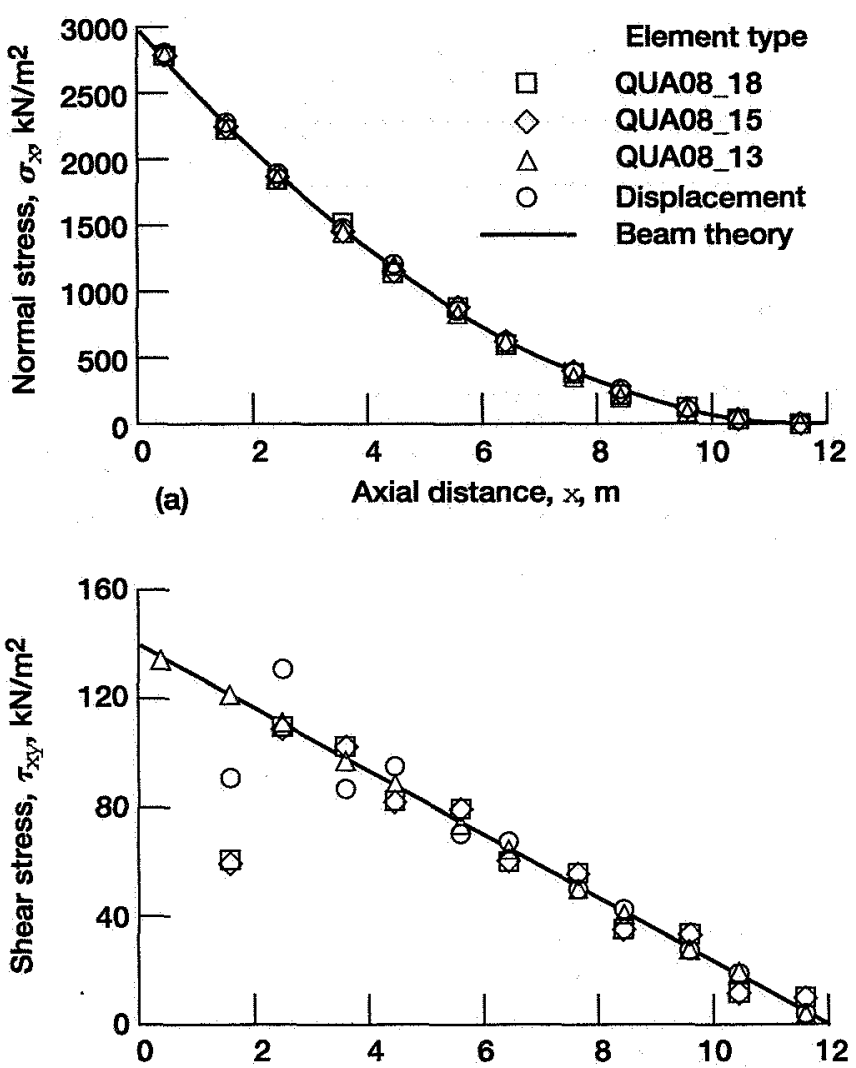

(b)

Axial distance, $\mathrm{x}, \mathrm{m}$

Figure 5.-Stress distribution along line $y=-y_{g}$ of cantilever beam subjected to uniformly distributed load. (a) Normal stress. (b) Shear stress.

obtained with element QUA08_13 are in excellent agreement with the corresponding analytical solutions. However, caution must be exercised in using this element because of the presence of spurious zero-energy modes.

\section{Example 2: Pure Bending of a Circular Arch}

A circular arch of radius $r_{o}$ and rectangular cross section $d$ by $H$, as shown in figure 6(a), is considered next. The arch is assumed to be clamped at $\theta=0^{\circ}$, loaded with a concentrated moment of intensity $M$ at $\theta=90^{\circ}$ (where $\theta$ is an angular coordinate), and made of a homogeneous and isotropic material with parameters $E$ and $v$. This example is presented with the specific purpose of demonstrating the validity of the present elements in modeling domains with curved boundaries. A state of plane stress was assumed, and the arch was modeled by using two-dimensional finite element discretizations, as shown in figure 6(b). The boundary conditions for the clamped end were the same as those applied to the cantilever beam in the previous example. The circles and asterisks shown in figure 6(b) have the same meaning as in Example 1. The nodes denoted by asterisks were not present when the arch was discretized with four-node 

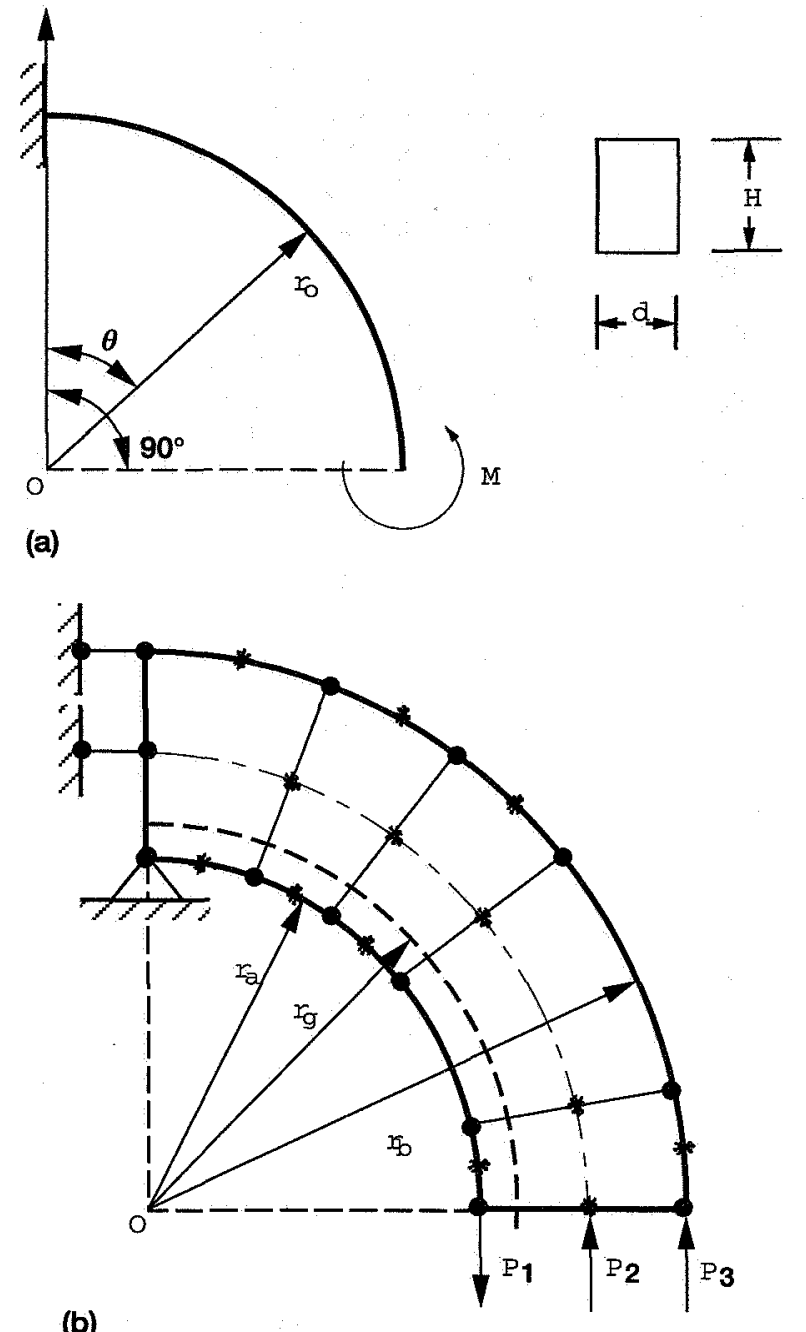

(b)

Figure 6.-A circular arch subjected to concentrated moment $M$. (a) One-dimensional model of a circular arch. (b) Two-dimensional finite element discretization.

quadrilateral elements. In the finite element discretization, radii $r_{a}$ and $r_{b}$ denote the inner and outer contour, respectively, with $r_{a}=r_{o}-0.5 \mathrm{H}$, and $r_{b}=r_{o}+0.5 \mathrm{H}$. The concentrated moment $M$ was modeled by using the concentrated forces of intensities $P_{1}, P_{2}$, and $P_{3}$, which correspond to the exact stress distribution due to the concentrated moment.

Let us first analyze the arch for $r_{a}=10 \mathrm{~m}$ and $r_{b}=12 \mathrm{~m}$. Such an arch may be characterized as a thin arch (ref. 4). The remaining parameters are taken to be $d=1.0 \mathrm{~m} ; E=21 \times 10^{7} \mathrm{kN} / \mathrm{m}^{2}$; $v=0.3$; and $M=600.0 \mathrm{kNm}$. The intensities of concentrated forces were calculated as $P_{1}=270.3 \mathrm{kN}, P_{2}=56.3 \mathrm{kN}$, and $P_{3}=326.6 \mathrm{kN}$ for discretizations with eight-node elements, and as $P_{1}=P_{2}=300 \mathrm{kN}$ for discretizations with four-node elements.

Results obtained with the present quadrilateral elements in a convergence study of the horizontal component $u$ of the tip displacement, along with those obtained with an eight-node displacement isoparametric element, are shown in figure 7. The tip displacements were normalized with respect to the exact solution, which was calculated from the plane stress theory (ref. 23). The present elements, especially those with a quadratic interpolation of geometry, performed well. Note that the results for element QUA04_05 were obtained with an element local coordinate system such that the local $x$-axis is defined by the element centroid and the center of one of the element sides. The results obtained with the local axes parallel to the global axes are not shown because the responses exhibited unstable oscillations. This behavior, which is due to representing the stress field in terms of incomplete polynomials, reveals the high sensitivity of element QUA04_05 to the orientation of local coordinate systems. It also demonstrates the benefits of employing stress field representations composed of complete polynomials in the analysis of general two-dimensional problems.

The displacements $u$ along the line $r=r_{o}$ were calculated next by using the discretization with six eight-node elements (see fig. 8). Exact displacements, calculated from the beam theory, are also shown in figure 8 for comparison. Again, the results are in good agreement.

Stress distributions for a circular arch were also calculated. The results for normal stresses $\sigma_{r}$ and $\sigma_{t}$ along the line $r=r_{g}=10.423 \mathrm{~m}$ are shown in figure 9; they are compared with

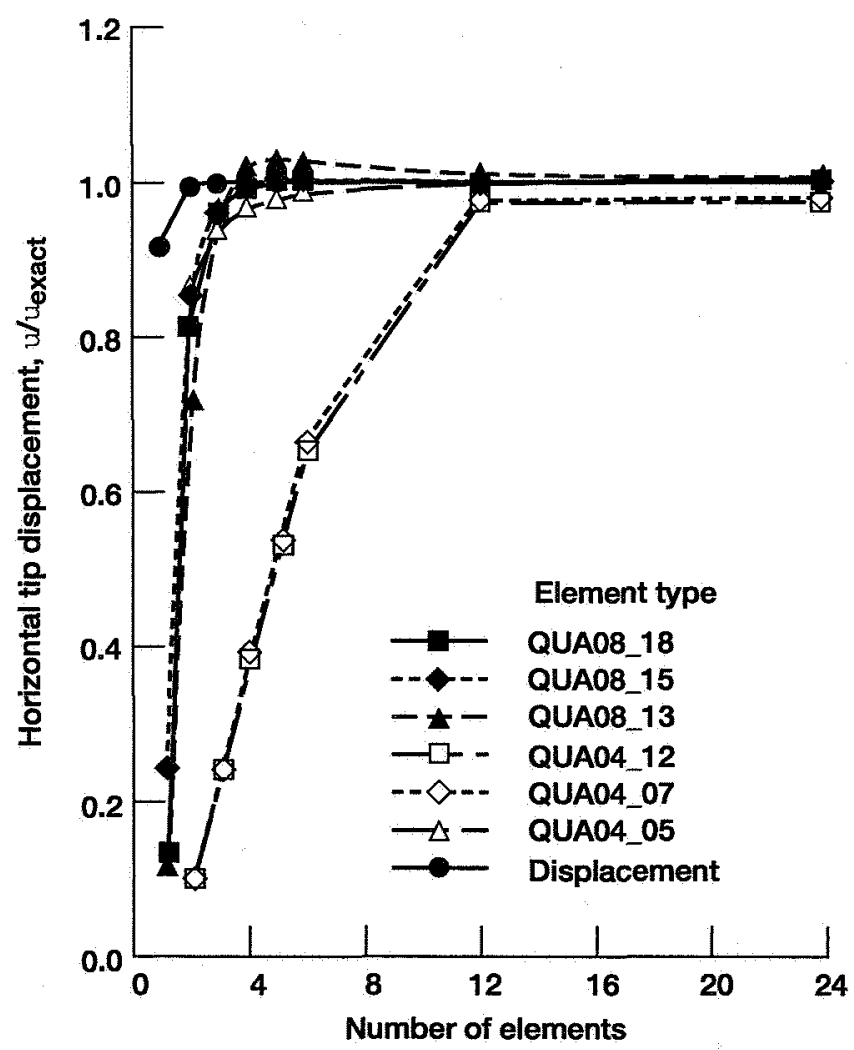

Figure 7.-Convergence study of tip displacement $u$ of a thin circular arch. 


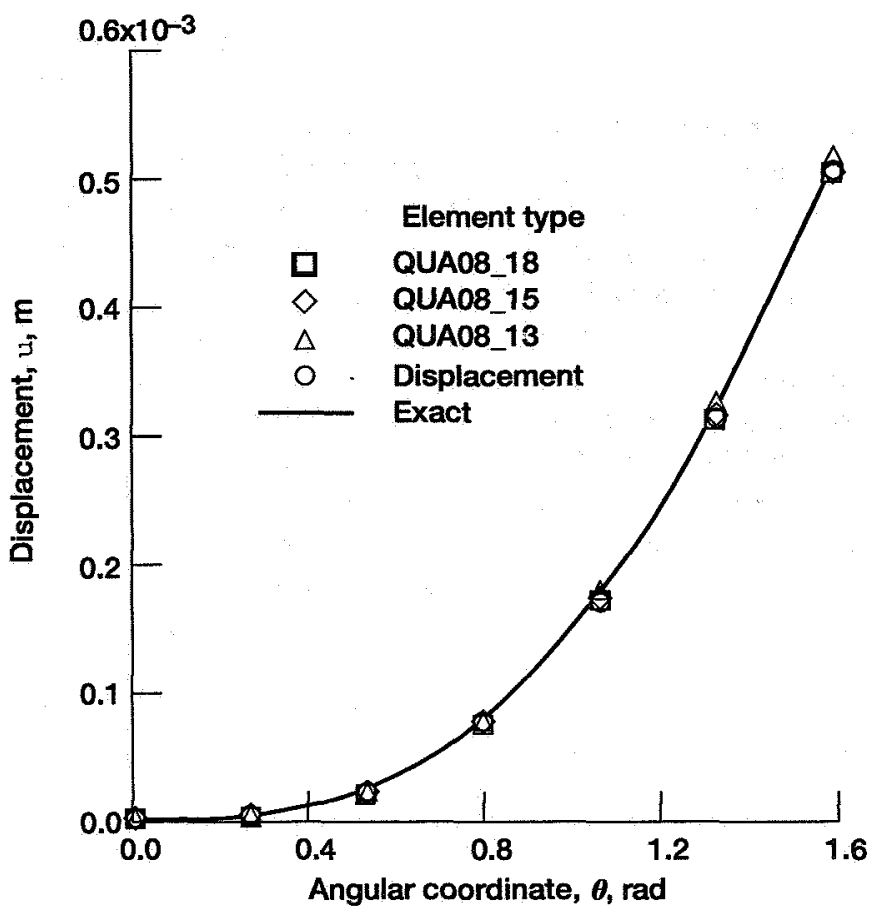

Figure 8.-Displacement distribution along line $r=r_{0}$ of thin circular arch.
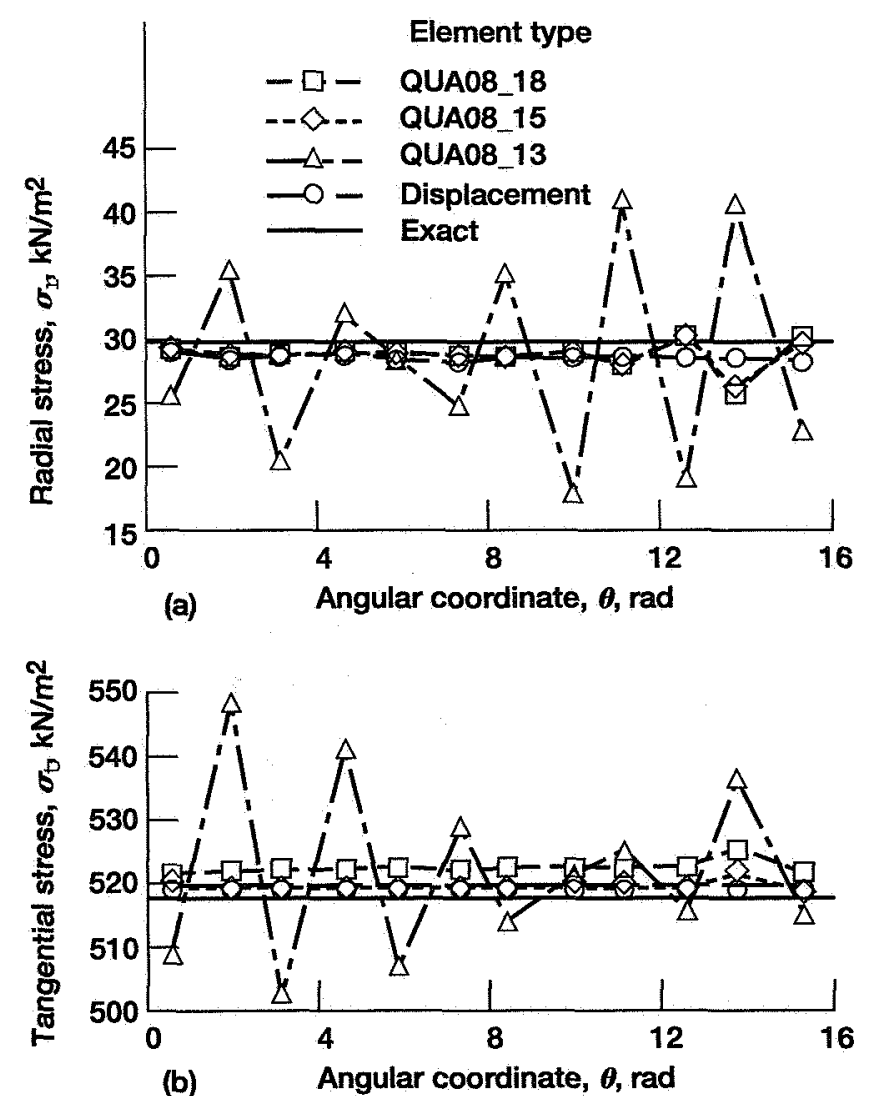

Figure 9.-Stress distribution along line $r=r_{g}$ of thin circular arch. (a) Radial stress. (b) Tangential stress.
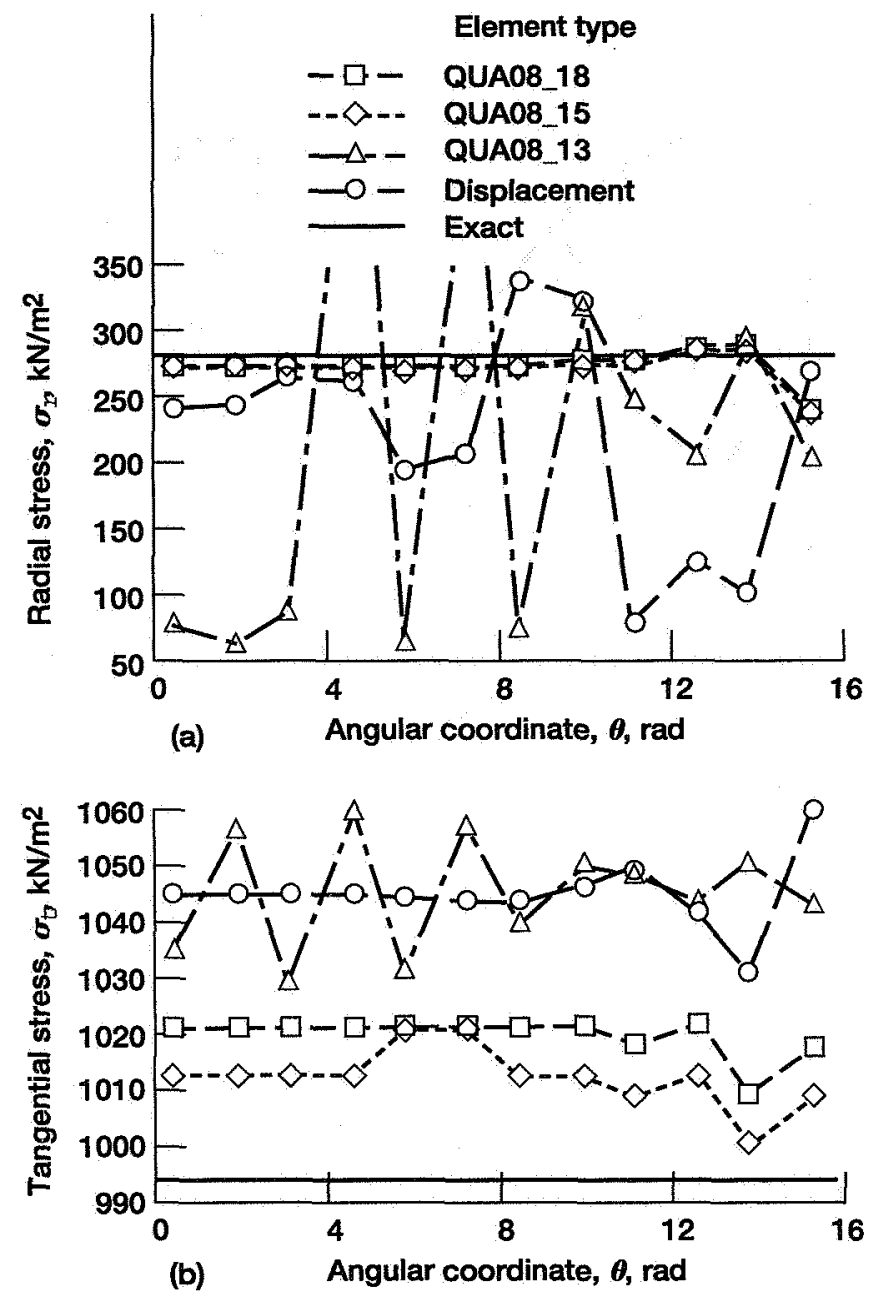

Figure 10.-Stress distribution along line $r=\Sigma_{\mathrm{g}}$ of a thick circular arch. (a) Radial stress. (b) Tangential stress.

corresponding solutions from the displacement formulation and with the exact solution (ref. 23). Elements QUA08_15 and QUA08_18 performed slightly better than the displacement element. Figure 9 also shows that element QUA08_13 does not provide good stress predictions in this case. The stress field used for this element was obviously constructed to exactly model the beam bending. However, it does not satisfy Navier's equations of equilibrium, and it does contain spurious zeroenergy modes for some configurations. These characteristics make element QUA08_13 unsuitable for modeling general problems of two-dimensional elasticity.

Stress distributions were also calculated for an arch with dimensions $r_{a}=1.0 \mathrm{~m}, r_{b}=2.0 \mathrm{~m}, r_{g}=1.211 \mathrm{~m}$, and $M=$ $300.0 \mathrm{kNm}$, with the remaining parameters being the same as before. Such an arch can be characterized as a thick arch. The concentrated forces used to model the moment $M$ for this case were $P_{1}=338.2 \mathrm{kN}, P_{2}=-73.1 \mathrm{kN}$, and $P_{3}=265.1 \mathrm{kN}$. Normal stresses $\sigma_{r}$ and $\sigma_{t}$ were calculated by using the present eight-node elements; the results, together with corresponding analytical 
solutions, are shown in figure 10. Note thatelements QUA08_15 and QUA08_18 provide more accurate results than the isoparametric displacement element, especially for radial stress $\sigma_{r}$, whereas element QUA08_13 exhibits the same problems as were observed for the thin arch. Also note that the reduced number of independent forces in element QUA08_15, as compared to element QUA08_18, did not lead to a loss of accuracy.

\section{Example 3: A Rectangular Plate Under Sinusoidal Load}

A rectangular plate with dimensions $2 L$ by $2 a$ is shown in figure 11 along with its support conditions. The plate is subjected to a vertical load of intensity $q(x)=q_{o} \sin (\pi x / 2 L)$. Since the geometry and the loading of the plate are symmetric with respect to the vertical axis, only half of the plate need be analyzed. The plate's finite element discretization using quadrilateral elements is also shown in figure 11. A state of plane stress was assumed for this analysis. The numerical values of the parameters were taken as $E=21.0 \times 10^{7} \mathrm{kN} / \mathrm{m}^{2}, v=0.3$, $L=10.0 \mathrm{~m}, a=5.0 \mathrm{~m}$; and $q_{o}=10.0 \mathrm{kN} / \mathrm{m}^{2}$. For comparison, the analytical solution of the problem was derived by following the procedure outlined in reference 23 ; this derivation is given in appendix $C$.

The stress response was calculated for locations lying along the line $A B$ by using both four- and eight-node quadrilateral elements, that is, with a mesh of $6 \times 6$ four-node elements and $4 \times 4$ eight-node elements, respectively. The stresses were first calculated for locations corresponding to the optimal sampling points for the displacement and hybrid methods. These locations correspond to Gauss integration points for $1 \times 1$ point integration when four-node elements are used, and $2 \times 2$ point integration when eight-node elements are used (ref. 24). Figures 12 and 13 show the results for four- and eight-node

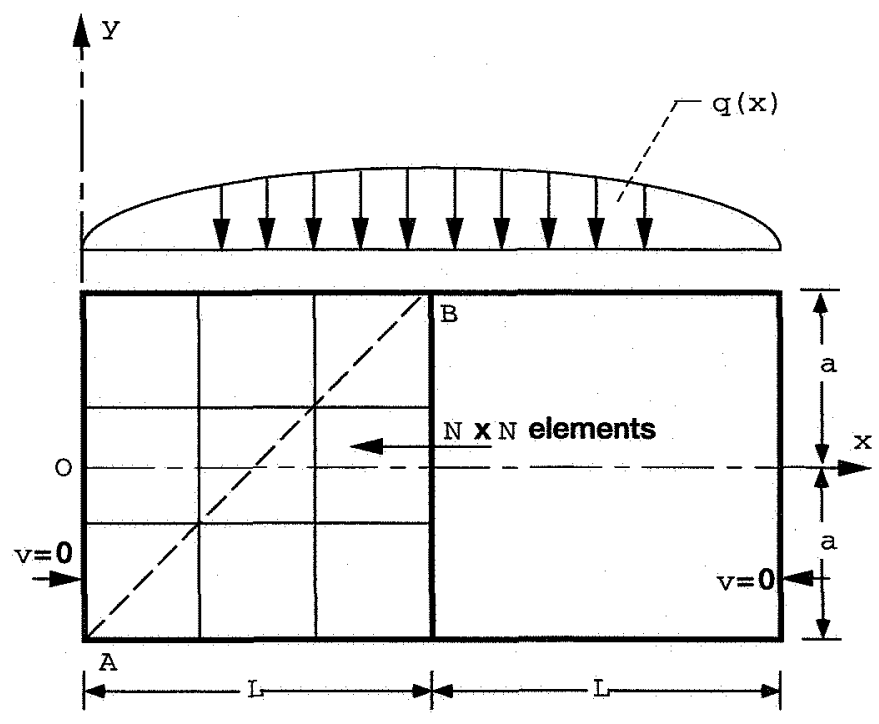

Figure 11. - A rectangular plate under sinusoidal loading. elements, respectively. These results agree well with the analytical solutions given in equations (C1), especially those for the eight-node elements. The present elements performed slightly better than the displacement formulation.

The influence of the location of sampling points on the accuracy of stress predictions was also investigated. The stresses were calculated by using eight-node elements at locations corresponding to $3 \times 3$ Gauss integration points. The results, shown in figure 14, indicate that the present elements are less sensitive to the location of sampling points than the corresponding displacement elements. It may also be concluded that the present elements provide better overall stress approximations within the element domains.
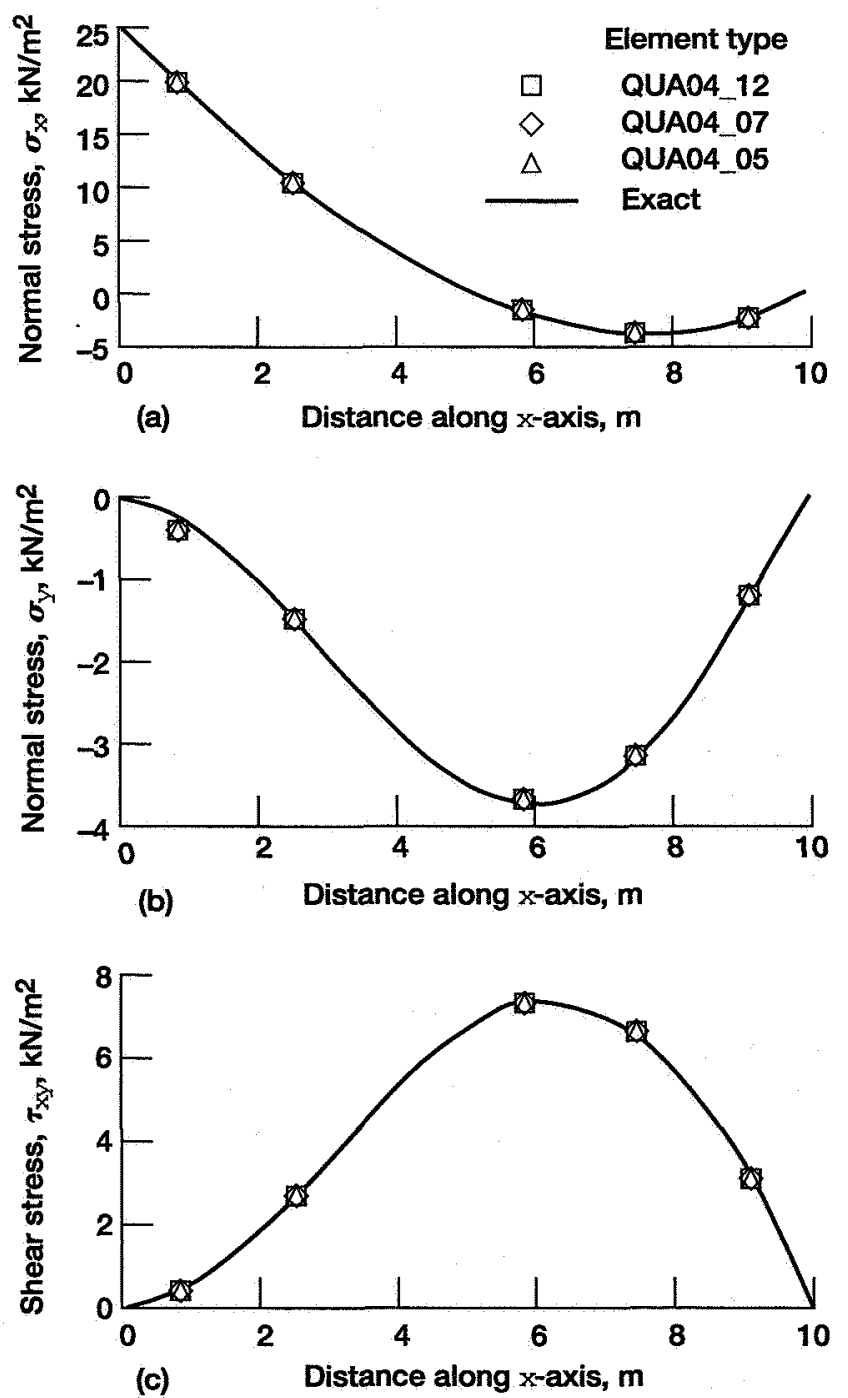

Figure 12.-Stress distribution along line $A B$ of a rectangular plate as determined by using four-node elements at 1-point Gauss integration locations. (a) Normal stress $\sigma_{\mathbf{x}}$ (b) Normal stress $\sigma_{y^{\prime}}(c)$ Shear stress $\tau_{\mathrm{XY}}$ 

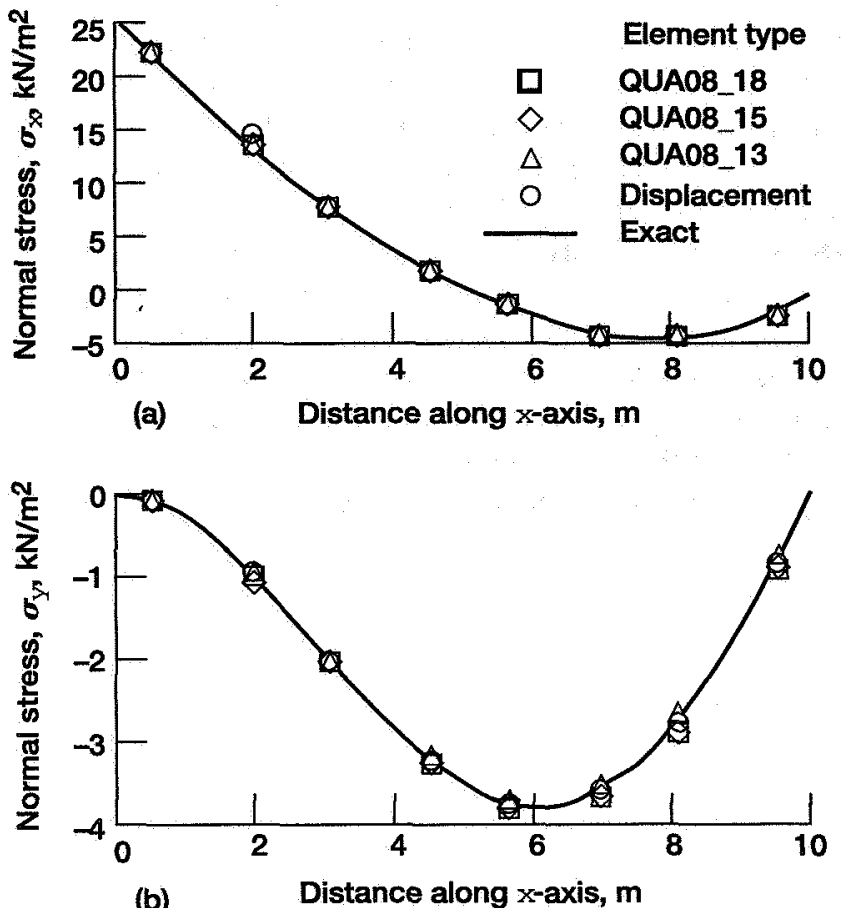

(b)

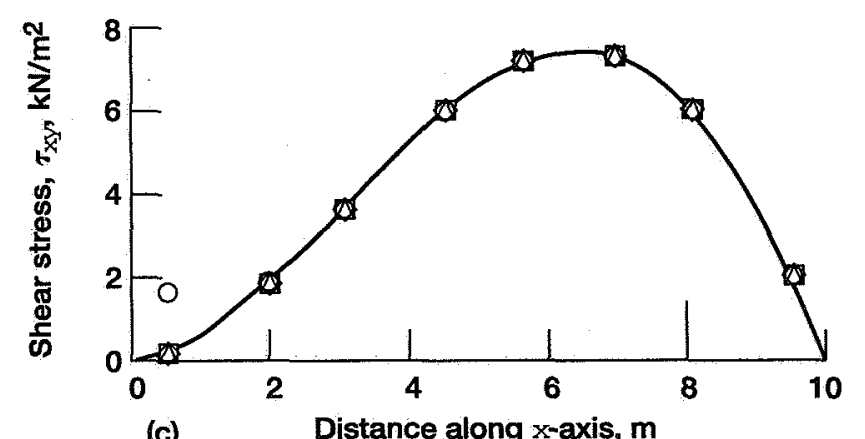

Figure 13. - Stress distribution along line $A B$ of a rectangular plate as determined by using eight-node elements at 2-point Gauss integration locations. (a) Normal stress $\sigma_{\mathrm{X}}$ (b) Normal stress $\sigma_{y^{*}}$ (c) Shear stress $\tau_{\mathrm{XY}}$

Displacement calculations for the plate were also performed. The horizontal component $u$ of displacement was calculated at element nodes lying along the line $x=0$. In figure 15 the results are compared with the exact solution given in equations (C2). A good agreement of the results is shown.

\section{Example 4: A Rectangular Plate With a Circular Hole Under Uniform Tension}

A rectangular plate of dimensions $2 a$ by $2 b$ with a circular hole of radius $r$, as shown in figure 16(a), was analyzed. The plate was assumed to be subjected to a uniform tension of intensity $q$ along the $O x$-axis and made of a homogeneous and isotropic material. Because of the double symmetry of the

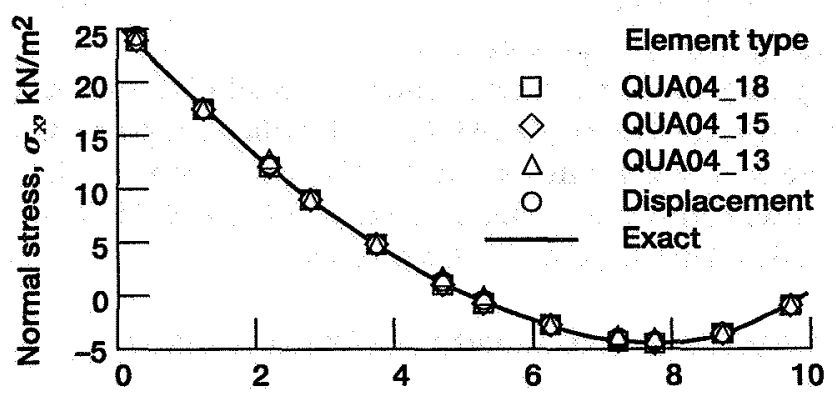

(a) Distance along $x$-axis, $m$
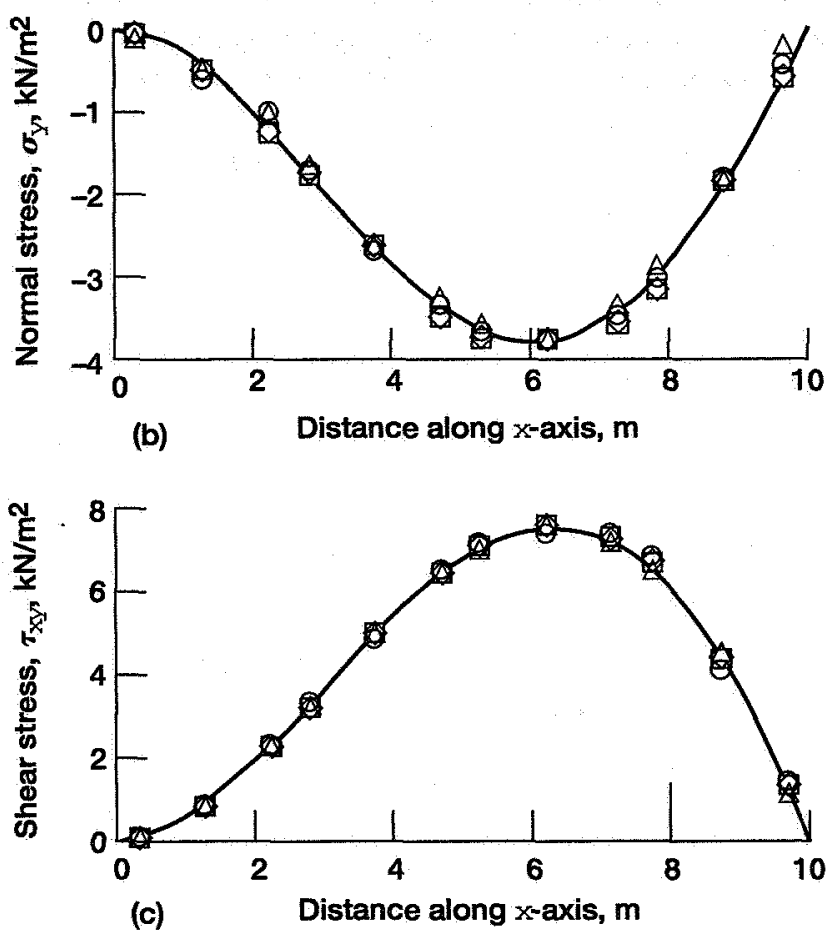

Figure 14.-Stress distribution along line $A B$ of a rectangular plate as determined by using eight-node elements at 3-point Gauss integration locations. (a) Normal stress $\sigma_{\mathrm{X}^{*}}$ (b) Normal stress $\sigma_{Y^{-}}$(c) Shear stress $\tau_{X Y^{*}}$

geometry and the loading of the plate, only a quarter of the plate (i.e., that bound by the arc $A B$ and lines $B C, C D, D E$, and $E A$ ) was discretized with finite elements. Finite element discretizations using quadrilateral and triangular elements are shown in figure 16, parts (b) and (c), respectively. The symmetry boundary conditions $u=0$ along the line $A E$, and $v=0$ along the line $B C$ were applied. A state of plane stress was assumed in the analysis.

The stress concentration factor at point $A$ of the plate was calculated for $a=48 \mathrm{~cm}, b=24 \mathrm{~cm}$, and $r=6 \mathrm{~cm}$ by using the entire element library. The results, given in table I, are compared with the adjusted stress concentration factor calculated by using the expression from reference 30 (i.e., $k=3.2126$ for the given dimensions of the plate). A very good agreement for 


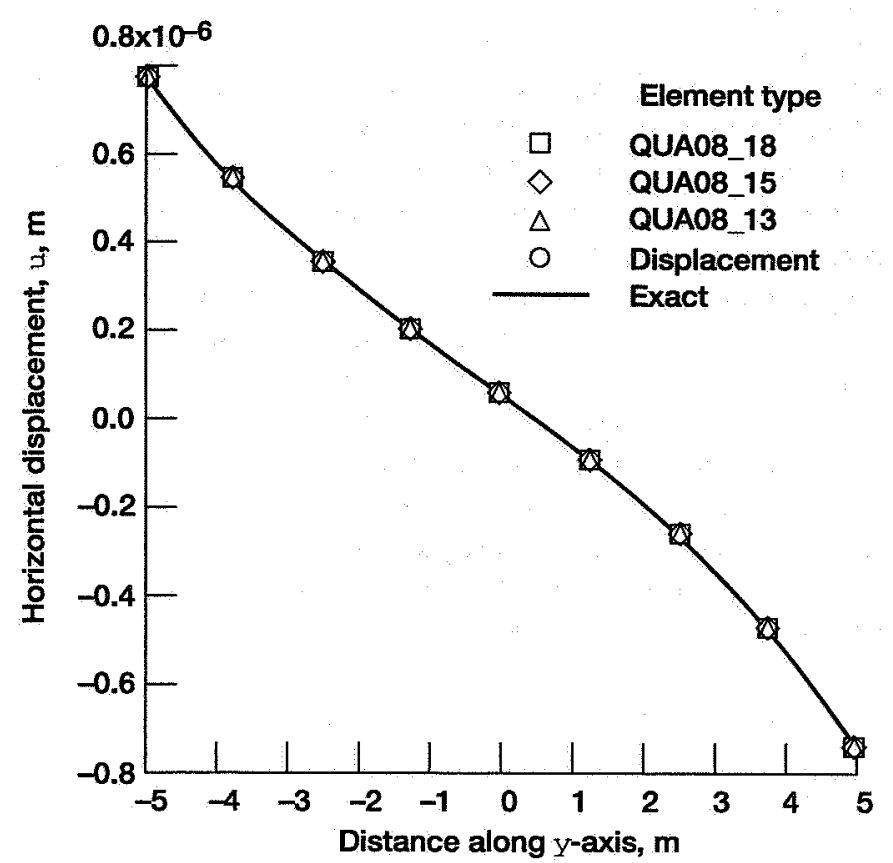

Figure 15.-Horizontal displacement along line $x=0$ of rectangular plate.

the stress concentration factor was achieved with the elements from the present library, especially with those with quadratic interpolation of geometry, as depicted in table I. This example further demonstrates the accuracy of the Integrated Force Method in stress calculations, particularly at locations that do not coincide with optimal sampling points.

\section{Discussion}

The validity and accuracy of the finite element library presented in this paper were demonstrated through numerical examples. Both one- and two-dimensional problems of elasticity were analyzed, and from the numerical results previously presented, a comparison can be made of the element performances. A careful examination of numerical results reveals that elements with quadratic interpolation of geometry performed better than those with linear interpolation of geometry. This is only partly due to the higher order of approximation of the stress fields, and thus, larger number of independent forces. Such a conclusion is supported by the fact that an increase in the number of independent forces in three-node triangular elements does not result in improved accuracy, and that higher order approximations of stress fields for four-node quadrilateral elements may result in overly rigid models. We may conclude that the interpolations of stress and displacement fields cannot be chosen arbitrarily, but must be compatible with stress-strain law.Moreover,elements QUA08_18 andQUA08_15exhibited better overall performance than element QUA08_13. The stress fields used for these two elements are represented by complete
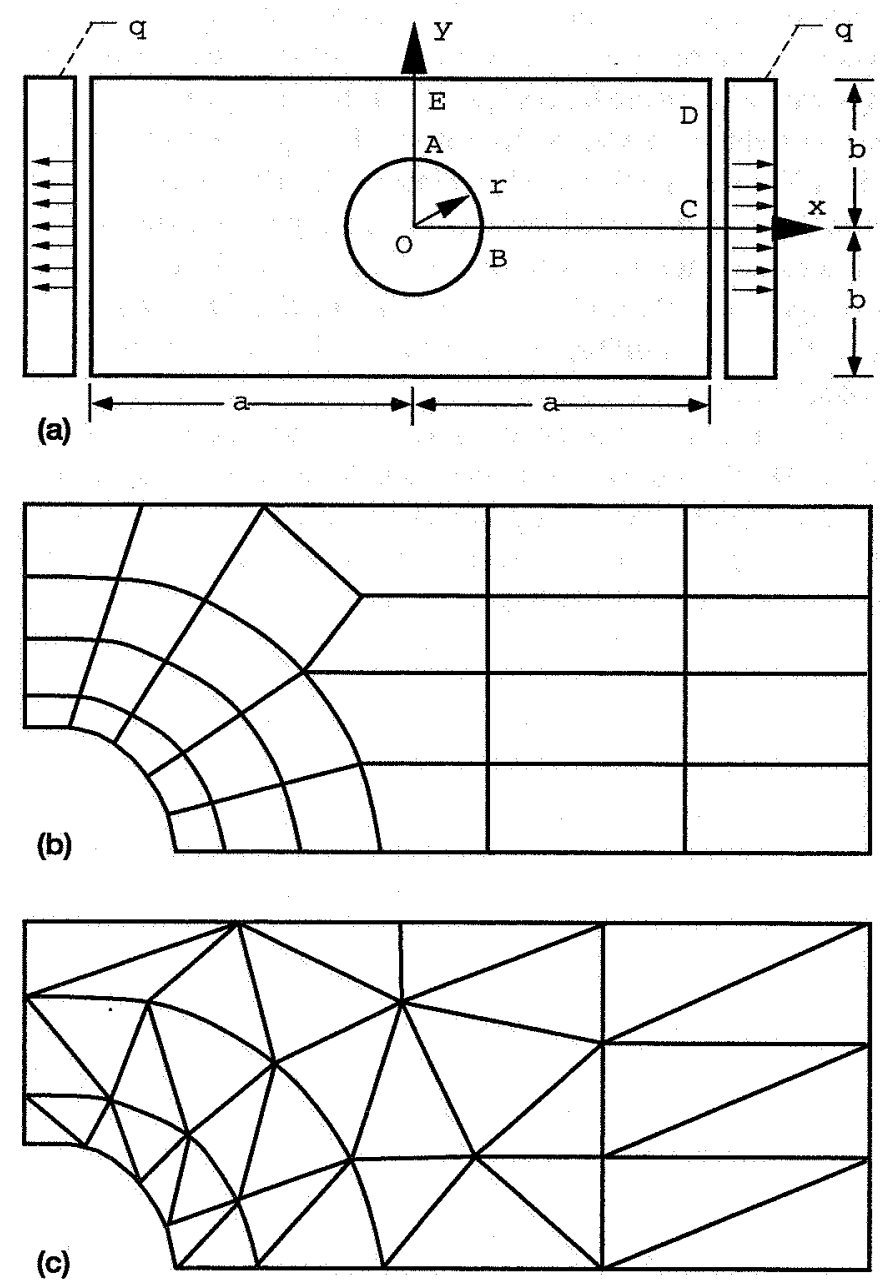

Figure 16.-Finite element discretizations of rectangular plate with circular hole in uniform tension. (a) Twodimensional plate. (b) Discretization with quadrilateral elements. (c) Discretization with triangular elements.

TABLE I.-STRESS CONCENTRATION FACTOR AT LOCATION A FOR THE PLATE WITH A HOLE

\begin{tabular}{|c|c|c|c|}
\hline $\begin{array}{c}\text { Element } \\
\text { type }\end{array}$ & $\begin{array}{c}\text { Number of } \\
\text { elements }\end{array}$ & $\begin{array}{c}\text { Number of force } \\
\text { unknowns }\end{array}$ & $\begin{array}{c}\text { Stress concentration } \\
\text { factor }\end{array}$ \\
\hline TRI03_03 & 37 & 111 & 2.54649 \\
TRI03_05 & & 185 & 2.54649 \\
TR103_07 & & 259 & 2.54649 \\
\hline TR106_09 & 37 & 333 & 3.25875 \\
TRI06_11 & & 407 & 3.15126 \\
TR106_12 & & 444 & 3.10322 \\
\hline QUA04_05 & 30 & 150 & 2.74766 \\
QUA04_07 & & 210 & 2.89305 \\
QUA04_12 & & 360 & 2.96215 \\
\hline QUA08_13 & 30 & 390 & 3.00314 \\
QUA08_15 & & 450 & 3.25266 \\
QUA08_18 & & 540 & 3.26137 \\
\hline
\end{tabular}


third-order polynomials, which produce elements that are invariant with respect to transformation of the local coordinate systems and that are free of spurious zero-energy modes. These stress fields also identically satisfy the equations of equilibrium. The stress field used for element QUA08_13, on the other hand, was represented by second-order polynomials, which yielded two spurious zero-energy modes for the rectangular configuration of the element. In addition, it did not satisfy the equations of equilibrium, and thus led to erroneous stress calculations in Example 2.

For some problems, good results were obtained with element QUA04_05, which has a very small number of unknown independent forces. The tip displacement convergence study in Example 2 revealed, however, that this element is sensitive to the orientation of coordinate axes and may not always be reliable in the analysis of domains with arbitrary geometric configurations. Moreover, element QUA04_05 provides accurate stresses only in the centroid, which may not always suffice. Elements with quadratic interpolation of geometry produce more accurate stress predictions at the optimal sampling points and at arbitrary locations within the element. Similar conclusions can be drawn for triangular elements.

The Integrated Force Method was also compared with the assumed displacement based finite element method. The results presented here reveal that the Integrated Force Method is better overall for stress calculations and provides displacement predictions of comparable accuracy.

\section{Concluding Remarks}

A finite element library was developed to analyze twodimensional structural mechanics problems by the Integrated Force Method. Triangular- and quadrilateral-shaped elements capable of modeling domains with arbitrary geometric configurations were presented. The displacement and stress fields were independently approximated. Displacement interpolation was performed as in the standard displacement method, and a procedure was developed to derive the stress interpolation functions in terms of complete polynomials of the required order. An Airy stress function was written as a complete polynomial of order $p$ that contains $p+1$ independent constants. The definitions of stress components in terms of stress functions were used next to derive the expressions for stresses. Elimination of the dependent constants from the expressions for stresses yielded stress fields expressed in terms of complete polynomials of order $p-2$. Stress fields thus defined identically satisfied the equations of equilibrium. The resulting element matrices had the correct rank and were insensitive to the transformation of local coordinate systems.

The present elements were applied to solve a variety of problems in two-dimensional elasticity. Comparisons were made with corresponding analytical solutions, and there was good agreement of the results. A series of numerical tests were performed in order to assess the relative performances of the present elements. These studies showed that elements with quadratic interpolations of geometry and displacements provide reliable predictions for all problems. The four-node quadrilateral elements performed well for some problems. Element QUA04_05 was shown to provide good results for a small number of unknown quantities, but it was sensitive to the orientation of the local coordinate system. This condition restricts its range of application.

The Integrated Force Method was also compared with the standard displacement method. The results presented here reveal that overall the Integrated Force Method performs better in stress calculations and exhibits an accuracy in displacement predictions comparable to the displacement method. These results confirm that the Integrated Force Method can be used successfully and efficiently in structural analysis and provide justification for efforts to incorporate the force method of analysis into general purpose finite element programs.

\author{
Lewis Research Center \\ Cleveland, Ohio \\ July 17, 1995
}




\section{Appendix A}

\section{Symbols}

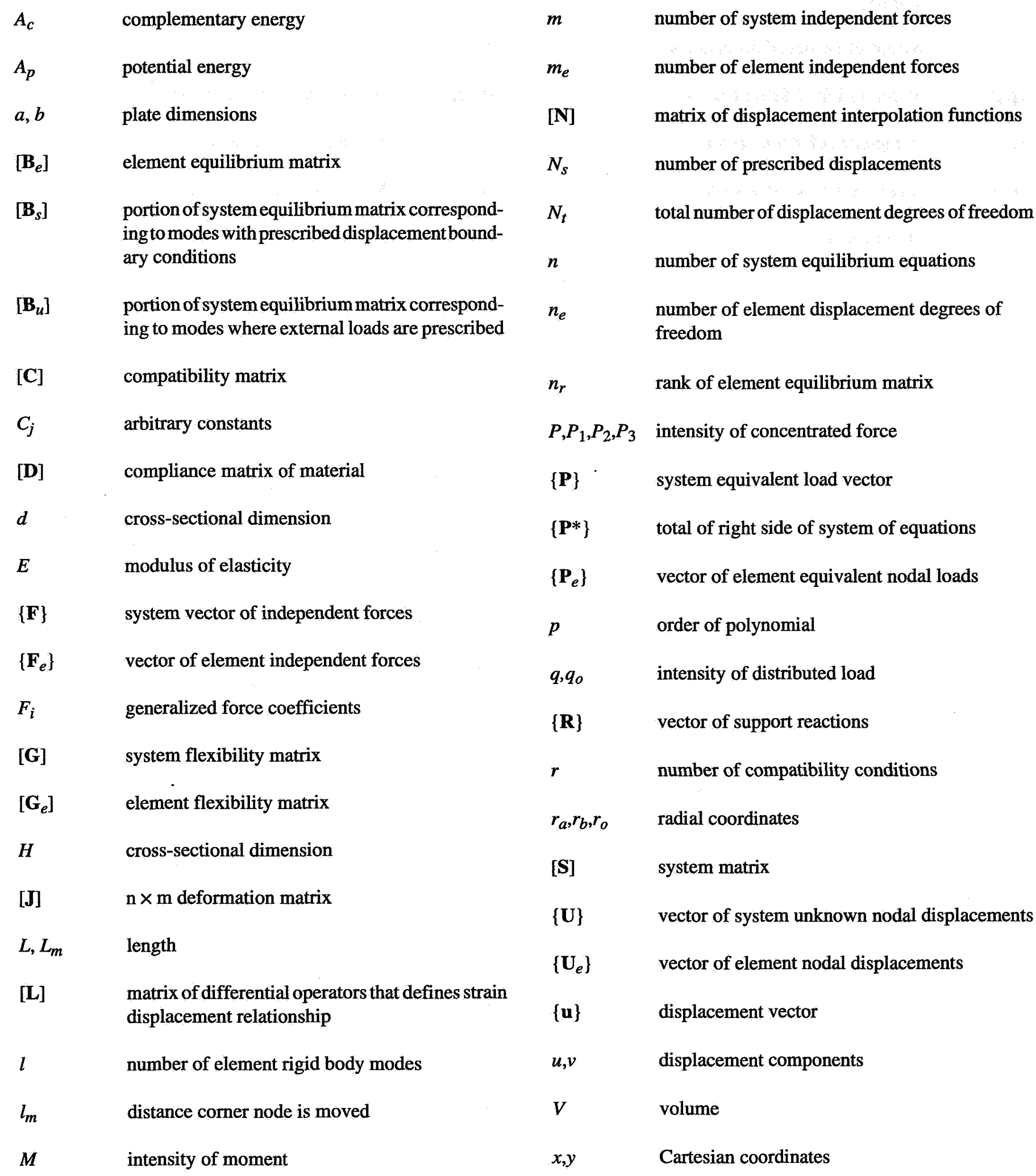




$\begin{array}{llll}{[\mathbf{Y}]} & \text { stress interpolation matrix } & \varepsilon_{x} \varepsilon_{y} & \text { components of strain vector } \\ y_{g} & \text { coordinate of Gauss point } & \theta & \text { angular coordinate } \\ {[\mathbf{Z}]} & {[\mathbf{L}][\mathbf{N}]} & v & \text { Poisson's ratio } \\ \left\{\beta_{e}\right\} & \text { vector of element deformations } & \{\sigma\} & \text { stress vector } \\ \left\{\beta_{o}\right\} & \text { vector of initial deformations } & \sigma_{x}, \sigma_{y} & \text { components of stress vector } \\ \gamma_{x y} & \text { component of strain vector } & \tau_{x y} & \text { component of stress vector } \\ \left\{\delta_{o}\right\} & \text { effective deformation vector } & \phi & \text { linear functions of constants } \\ \{\varepsilon\} & \text { strain vector } & \Phi & \text { Airy stress function }\end{array}$




\section{Appendix B}

\section{Expressions for Stress Fields}

(a) Full Polynomials Derived from the Stress Function

- constant terms:

$$
\begin{aligned}
& \sigma_{x}^{(0)}=F_{1}^{(0)} \\
& \sigma_{y}^{(0)}=F_{2}^{(0)} \\
& \tau_{x y}^{(0)}=F_{3}^{(0)}
\end{aligned}
$$

- linear terms:

$$
\begin{aligned}
\sigma_{x}^{(1)} & =F_{1}^{(1)} x+F_{2}^{(1)} y \\
\sigma_{y}^{(1)} & =F_{3}^{(1)} x+F_{4}^{(1)} y \\
\tau_{x y}^{(1)} & =-F_{4}^{(1)} x-F_{1}^{(1)} y
\end{aligned}
$$

- quadratic terms:

$$
\begin{aligned}
\sigma_{x}^{(2)} & =-\frac{1}{2} F_{5}^{(2)} x^{2}+F_{1}^{(2)} y^{2}+F_{2}^{(2)} x y \\
\sigma_{y}^{(2)} & =F_{3}^{(2)} x^{2}-\frac{1}{2} F_{5}^{(2)} y^{2}+F_{4}^{(2)} x y \\
\tau_{x y}^{(2)} & =-\frac{1}{2} F_{4}^{(2)} x^{2}-\frac{1}{2} F_{2}^{(2)} y^{2}+F_{5}^{(2)} x y
\end{aligned}
$$

- cubic terms:

$$
\begin{aligned}
& \sigma_{x}^{(3)}=-\frac{1}{3} F_{5}^{(3)} y^{3}+F_{1}^{(3)} y^{3}-F_{6}^{(3)} x^{2} y+F_{2}^{(3)} x y^{2} \\
& \sigma_{y}^{(3)}=F_{3}^{(3)} x^{3}-\frac{1}{3} F_{6}^{(3)} y^{3}+F_{4}^{(3)} x^{2} y-F_{5}^{(3)} x y^{2} \\
& \tau_{x y}^{(3)}=-\frac{1}{3} F_{4}^{(3)}-\frac{1}{3} F_{2}^{(3)}+F_{5}^{(3)} x^{2} y+F_{6}^{(3)} x y^{2}
\end{aligned}
$$

- full cubic polynomial

$$
\begin{aligned}
\sigma_{x}= & F_{1}+F_{4} x+F_{5} y-\frac{1}{2} F_{12} x^{2}+F_{8} y^{2}+F_{9} x y- \\
& \frac{1}{3} F_{17} x^{3}+F_{13} y^{3}-F_{18} x^{2} y+F_{14} x y^{2} \\
\sigma_{y}= & F_{2}+F_{6} x+F_{7} y-F_{10} x^{2}+\frac{1}{2} F_{12} y^{2}+F_{11} x y+ \\
& F_{15} x^{3}-\frac{1}{3} F_{18} y^{3}+F_{16} x^{2} y-F_{17} x y^{2} \\
\tau_{x y}= & F_{3}-F_{7} x-F_{4} y-\frac{1}{2} F_{11} x^{2}-\frac{1}{2} F_{9} y^{2}+F_{12} x y- \\
& \frac{1}{3} F_{16} x^{3}-\frac{1}{3} F_{14} y^{3}+F_{17} x^{2} y+F_{18} x y^{2}
\end{aligned}
$$


(b) Full Polynomials Reduced Using the Condition $\nabla^{2}\left(\sigma_{x}+\sigma_{y}\right)=0$

- quadratic terms:

$$
\begin{aligned}
& \sigma_{x}^{(2 r)}=F_{1}^{(2 r)}\left(y^{2}-\frac{1}{2} x^{2}\right)+F_{2}^{(2 r)} x y-\frac{1}{2} F_{3}^{(2 r)} x^{2} \\
& \sigma_{y}^{(2 r)}=-\frac{1}{2} F_{1}^{(2 r)} y^{2}+F_{3}^{(2 r)}\left(x^{2}-\frac{1}{2} y^{2}\right)+F_{4}^{(2 r)} x y \\
& \tau_{x y}^{(2 r)}=F_{1}^{(2 r)} x y-\frac{1}{2} F_{2}^{(2 r)} y^{2}+F_{3}^{(2 r)} x y-\frac{1}{2} F_{4}^{(2 r)} x^{2}
\end{aligned}
$$

- cubic terms:

$$
\begin{aligned}
& \sigma_{x}^{(3 r)}=F_{1}^{(3 r)}\left(y^{3}-\frac{3}{2} x^{2} y\right)+F_{2}^{(3 r)}\left(x y^{2}-\frac{1}{6} x^{3}\right)-\frac{1}{2} F_{3}^{(3 r)} x^{3}-\frac{1}{2} F_{4}^{(3 r)} x^{2} y \\
& \sigma_{y}^{(3 r)}=-\frac{1}{2} F_{1}^{(3 r)} y^{3}-\frac{1}{2} F_{2}^{(3 r)} x y^{2}+F_{3}^{(3 r)}\left(x^{3}-\frac{3}{2} x y^{2}\right)+F_{4}^{(3 r)}\left(x^{2} y-\frac{1}{6} y^{3}\right) \\
& \tau_{x y}^{(3 r)}=\frac{3}{2} F_{1}^{(3 r)} x y^{2}+F_{2}^{(3 r)}\left(\frac{1}{2} x^{2} y-\frac{1}{3} y^{3}\right)+\frac{3}{2} F_{3}^{(3 r)} x^{2} y+F_{4}^{(3 r)}\left(\frac{1}{2} x y^{2}-\frac{1}{3} x^{3}\right)
\end{aligned}
$$

(c) Stress Field Used for the Element QUA04_05

$$
\begin{aligned}
\sigma_{x} & =F_{1}+F_{4} y \\
\sigma_{y} & =F_{2}+F_{5} x \\
\tau_{x y} & =F_{3}
\end{aligned}
$$

(d) Stress Field Used for the Element TRI06_09

$$
\begin{aligned}
\sigma_{x} & =F_{1}+F_{2} y+F_{6} x-2 F_{8} x y \\
\sigma_{y} & =F_{3}+F_{4} x+F_{7} y-2 F_{9} x y \\
\tau_{x y} & =F_{5}-F_{6} y-F_{7} x+F_{8} y^{2}+F_{9} x^{2}
\end{aligned}
$$

(e) Stress Field Used for the Element QUA08_13

$$
\begin{aligned}
\sigma_{x} & =F_{1}+F_{2} y+F_{6} x+F_{8} y^{2}+F_{10} x^{2}-2 F_{13} x y \\
\sigma_{y} & =F_{3}+F_{4} x+F_{7} y+F_{9} x^{2}+F_{11} y^{2}-2 F_{12} x y \\
\tau_{x y} & =F_{5}-F_{6} y-F_{7} x-2\left(F_{10}+F_{11}\right) x y+F_{12} x^{2}+F_{13} y^{2}
\end{aligned}
$$




\section{Appendix C}

\section{Analytical Solution for the Rectangular Plate Under Sinusoidal Load}

The analytical solution of the problem presented in Example 3 is given here. It can be derived by using the Fourier series outlined in reference 23 . The stress components $\sigma_{x}, \sigma_{y}$, and $\tau_{x y}$ are given as

$\sigma_{x}=\alpha^{2}\left[C_{1} \cosh \alpha y+C_{2} \sinh \alpha y+C_{3}\left(\frac{2}{\alpha} \sinh \alpha y+y \cosh \alpha y\right)\right.$

$$
\left.+C_{4}\left(\frac{2}{\alpha} \cosh \alpha y+y \sinh \alpha y\right)\right] \sin \frac{\pi x}{2 L}
$$

$\sigma_{y}=-\alpha^{2}\left(C_{1} \cosh \alpha y+C_{2} \sinh \alpha y+C_{3} y \cosh \alpha y\right.$

$$
\left.+C_{4} y \cosh \alpha y\right) \sin \frac{\pi x}{2 L}
$$

$$
\begin{array}{r}
\tau_{x y}=\alpha^{2}\left[C_{1} \sinh \alpha y+C_{2} \cosh \alpha y+C_{3}\left(\frac{1}{\alpha} \cosh \alpha y+y \sinh \alpha y\right)\right. \\
\left.+C_{4}\left(\frac{1}{\alpha} \sinh \alpha y+y \cosh \alpha y\right)\right] \cos \frac{\pi x}{2 L} \quad \text { (C1c) }
\end{array}
$$

where $C_{1}=p_{0}(\sinh \alpha a+\alpha a \cosh \alpha a) / D_{1} ; C_{2}=-p_{0}(\cosh \alpha a+$ $\alpha a \sinh \alpha a) / D_{2} ; C_{3}=p_{0} \alpha \cosh \alpha a / D_{2} ;$ and $C_{4}=-p_{0} \alpha \sinh \alpha a l$ $D_{1}$; with $D_{1}=\alpha^{2}(2 \alpha a+\sinh 2 \alpha a), D_{2}=\alpha^{2}(2 \alpha a-\sinh 2 \alpha a)$, $\alpha=\pi / 2 L$, and $x$ and $y$ are coordinates of the point inside the plate as defined in figure 11 .

The displacement $u$ is calculated by integrating the straindisplacement relations as follows:

$$
u=-\frac{\alpha}{E}\left[C_{1}(1+v) \cosh \alpha y+C_{2}(1+v) \sinh \alpha y+C_{3}\right.
$$

$$
\left(\frac{2}{\alpha} \sinh \alpha y+(1+v) y \cosh \alpha y\right)
$$

$$
\left.+C_{4}\left(\frac{2}{\alpha} \cosh \alpha y+(1+v) y \sinh \alpha y\right)\right] \cos \frac{\pi x}{2 L}
$$




\section{References}

1. Zienkiewicz, O.C.: The Finite Element Method. McGraw-Hill Book Co., New York, 1977.

2. Cook, R.D.: Concepts and Applications of Finite Element Analysis. John Wiley \& Sons, New York, 1981.

3. Gallagher, R.H.: Finite Element Analysis: Fundamentals. Prentice-Hall, Inc., Englewood Cliffs, NJ, 1975.

4. Spilker, R.L.; Maskeri, S.M.; and Kania, E.: Plane Isoparametric HybridStress Elements: Invariance and Optimal Sampling. Int. J. Num. Meth. Engng., vol. 17, 1981, pp. 1469-1496.

5. Kaneko, I.; Lawo, M.; and Thierauf, G.: On Computational Procedures for the Force Method. Int. J. Num. Meth. Engng., vol. 18, 1982, pp. 1469-1495.

6. Pian, T.H.H.: Derivation of Element Stiffness Matrices by Assumed Stress Distributions. AIAA J., vol. 2, July 1964, pp. 1333-1336.

7. Pian, T.H.H.; and Tong, P.: Basis of Finite Element Methods for Solid Continua. Int. J. Num. Meth. Engng., vol. 1, 1969, pp. 3-28.

8. Pian, T.H.H.: A Historical Note About 'Hybrid Elements'. Int. J. Num. Meth. Engng., vol. 12, 1978, pp. 891-892.

9. Przemieniecki, J.S.: Theory of Matrix Structural Analysis. Dover Publications, Inc., New York, 1985.

10. Martin, H.C.: Introduction to Matrix Methods of Structural Analysis. McGraw-Hill Book Co., New York, 1966.

11. Robinson, J.: Integrated Theory of Finite Element Methods. John Wiley \& Sons, London, 1973.

12. Patnaik, S.N.: An Integrated Force Method for Discrete Analysis. Int. J. Num. Meth. Engng., vol. 6, 1973, pp. 237-251.

13. Patnaik, S.N.: The Integrated Force Method Versus the Standard Force Method. Comp. Structures, vol. 22, no. 2, 1986, pp. 151-163.

14. Patnaik, S.N.; Berke, L.; and Gallagher, R.H.: Integrated Force Method Versus Displacement Method for Finite Element Analysis. Comput. Struct., vol. 38, no. 4, 1991, pp. 377-407.

15. Patnaik, S.N.: The Variational Energy Formulation for the Integrated Force Method. AIAA J., vol. 24, no. 1, 1986, pp. 129-137.

16. Patnaik, S.N.; and Joseph, K.T.: Compatibility Conditions From Deformation Displacement Relationship. AIAA J., vol. 23, Aug. 1985, pp. 1291-1293.
17. Patnaik, S.N.; and Joseph, K.T.: Generation of the Compatibility Matrix in the Integrated Force Method. Comput. Meth. App. Mech. Engng., vol. 55, no. 3,1986 , pp. 239-257

18. Nagabhushanam, J; and Patnaik, S.N.: General Purpose Program to Generate Compatibility Matrix for the Integrated Force Method. AIAA J., vol. 28, Oct. 1990 , pp. 1838-1842.

19. Patnaik, S.N.; Berke, L.; and Gallagher, R.H.: Compatibility Conditions for Structural Mechanics for Finite Element Analysis. AIAA J., vol. 29, May 1991, pp. 820-829.

20. Patnaik, S.N., et al.: Improved Accuracy for Finite Element Structural Analysis Via a New Integrated Force Method. NASA TP-3204, 1992.

21. Patnaik, S.N.; and Yadagiri, S.: Frequency Analysis of Structures by Integrated Force Method. J. Sound Vib., vol. 83, no. 1, 1982, pp. 93-109.

22. Patnaik, S.N.; and Gallagher, R.H.: Gradients of Behaviour Constraints and Reanalysis Via the Integrated Force Method. Int. J. Num. Meth. Engng., vol. 23, 1986, pp. 2205-2212.

23. Timoshenko, S.P.; and Goodier, J.N.: Theory of Elasticity. McGraw-Hill Book Co., New York, 1970.

24. Spilker, R.L.; and Singh, S.P.: Three-Dimensional Hybrid-Stress Isoparametric Quadratic Displacement Elements. Int. J. Num. Meth. Engng., vol. 18, 1982, pp. 445-465.

25. Tong, P.; and Pian, T.H.H.: A Variational Principle and the Convergence of a Finite-Element Method Based on Assumed Stress Distribution. Int. J. Solids Struct., vol. 5, 1969, pp. 463-472.

26. Pian, T.H.H.; and Chen, D.: On the Suppression of Zero Energy Deformation Modes. Int. J. Num. Meth. Engng., vol. 19, 1983, pp. 1741-1752.

27. Ergatoudis, I.; Irons, B.M.; and Zienkiewics, O.C.: Curved, Isoparametric, "Quadrilateral" Elements for Finite Element Analysis. Int. J. Solids and Struct., vol. 4, 1968, pp. 31-42.

28. Dunavant, D.A.: High Degree Efficient Symmetrical Gaussian Quadrature Rules for the Triangle. Int. J. Num. Meth. Engng., vol. 21, 1985, pp. 1129-1148.

29. MacNeal, R.H.; and Harder, R.L.: A Proposed Standard Set of Problems to Test Finite Element Accuracy. Finite Elem Anal. Des., vol. 1, Apr. 1985, pp. 3-20.

30. Roark, R.J.; and Young, W.C.: Formulas for Stress and Strain. McGrawHill Book Co., New York, 1975. 
Public reporting burden for this collection of information is estimated to average 1 hour per response, including the time for reviewing instructions, searching existing data sources, gathering and maintaining the data needed, and completing and reviewing the collection of information. Send comments regarding this burden estimate or any other aspect of this collection of information, including suggestions for reducing this burden, to Washington Headquarters Services, Directorate for Intormation Operations and Reports, 1215 Jeflen
Davis Highway, Suite 1204, Arlington, VA 22202-4302, and to the Office of Management and Budget, Paperwork Reduction Project (0704-0188), Washington, DC 20503.
1. AGENCY USE ONLY (Leave blank) 2. REPORT DATE
March 1996
3. REPORT TYPE AND DATES COVERED
Technical Memorandum

\section{TITLE AND SUBTITLE}

Development of Finite Elements for Two-Dimensional Structural Analysis Using the Integrated Force Method

5. FUNDING NUMBERS

6. AUTHOR(S)

WU-505-63-53

Igor Kaljevic', Surya N. Patnaik, and Dale A. Hopkins

7. PERFORMING ORGANIZATION NAME(S) AND ADDRESS(ES)

8. Performing organization REPORT NUMBER

National Aeronautics and Space Administration

Lewis Research Center

Cleveland, Ohio 44135-3191

E-9240

9. SPONSORING/MONITORING AGENCY NAME(S) AND ADDRESS(ES)

10. SPONSORINGMONITORING AGENCY REPORT NUMBER

National Aeronautics and Space Administration

Washington, D.C. 20546-0001

NASA TM-4655

\section{SUPPLEMENTARY NOTES}

Igor Kaljevic and Surya N. Patnaik, Ohio Aerospace Institute, 22800 Cedar Point Road, Cleveland, Ohio 44142; Dale A. Hopkins, NASA Lewis Research Center. Responsible person, Dale A. Hopkins, organization code 5210, (216) 433-3260.

12a. DISTRIBUTION/AVAILABILITY STATEMENT

12b. DISTRIBUTION CODE

Unclassified - Unlimited

Subject Category 39

This publication is available from the NASA Center for Aerospace Information, (301) 621-0390.

\section{ABSTRACT (Maximum 200 words)}

The Integrated Force Method has been developed in recent years for the analysis of structural mechanics problems. This method treats all independent internal forces as unknown variables that can be calculated by simultaneously imposing equations of equilibrium and compatibility conditions. In this paper a finite element library for analyzing two-dimensional problems by the Integrated Force Method is presented. Triangular- and quadrilateral-shaped elements capable of modeling arbitrary domain configurations are presented. The element equilibrium and flexibility matrices are derived by discretizing the expressions for potential and complementary energies, respectively. The displacement and stress fields within the finite elements are independently approximated. The displacement field is interpolated as it is in the standard displacement method, and the stress field is approximated by using complete polynomials of the correct order. A procedure that uses the definitions of stress components in terms of an Airy stress function is developed to derive the stress interpolation polynomials. Such derived stress fields identically satisfy the equations of equilibrium. Moreover, the resulting element matrices are insensitive to the orientation of local coordinate systems. A method is devised to calculate the number of rigid body modes, and the present elements are shown to be free of spurious zero-energy modes. A number of example problems are solved by using the present library, and the results are compared with corresponding analytical solutions and with results from the standard displacement finite element method. The Integrated Force Method not only gives results that agree well with analytical and displacement method results but also outperforms the displacement method in stress calculations.

\section{SUBJECT TERMS}

Integrated Force Method; Two-dimensional analysis; Element library

17. SECURITY CLASSIFICATION OF REPORT Unclassified

\section{SECURITY CLASSIFICATION OF THIS PAGE Unclassified}

\section{SECURITY CLASSIFICATION OF ABSTRACT Unclassified}

A03 